\title{
Resolving the fragmentation of high line-mass filaments with ALMA: the integral shaped filament in Orion $A^{\star}$
}

\author{
J. Kainulainen ${ }^{1}$, A. M. Stutz ${ }^{1,2}$, T. Stanke ${ }^{3}$, J. Abreu-Vicente ${ }^{1}$, H. Beuther ${ }^{1}$, T. Henning ${ }^{1}$, \\ K. G. Johnston ${ }^{4}$, and S. T. Megeath ${ }^{5}$ \\ 1 Max-Planck-Institute for Astronomy, Königstuhl 17, 69117 Heidelberg, Germany \\ e-mail: jtkainul@mpia.de \\ 2 Departamento de Astronomía, Universidad de Concepción, Av. Esteban Iturra s/n, 160-C Distrito Universitario, Chile \\ 3 ESO, Karl-Schwarzschild-Strasse 2, 85748 Garching bei München, Germany \\ ${ }^{4}$ School of Physics \& Astronomy, E.C. Stoner Building, The University of Leeds, Leeds LS2 9JT, UK \\ 5 Ritter Astrophsical Research Center, Department of Physics and Astronomy, University of Toledo, Toledo, OH 43606, USA
}

Received 10 March 2016 / Accepted 26 January 2017

\begin{abstract}
We study the fragmentation of the nearest high line-mass filament, the integral shaped filament (ISF, line-mass $\sim 400 M_{\odot} \mathrm{pc}^{-1}$ ) in the Orion A molecular cloud. We have observed a $1.6 \mathrm{pc}$ long section of the ISF with the Atacama Large Millimetre/submillimeter Array (ALMA) at $3 \mathrm{~mm}$ continuum emission, at a resolution of $\sim 3^{\prime \prime}$ (1200 AU). We identify from the region 43 dense cores with masses about a solar mass. $60 \%$ of the ALMA cores are protostellar and $40 \%$ are starless. The nearest neighbour separations of the cores do not show a preferred fragmentation scale; the frequency of short separations increases down to 1200 AU. We apply a twopoint correlation analysis on the dense core separations and show that the ALMA cores are significantly grouped at separations below $\sim 17000 \mathrm{AU}$ and strongly grouped below $\sim 6000 \mathrm{AU}$. The protostellar and starless cores are grouped differently: only the starless cores group strongly below $\sim 6000 \mathrm{AU}$. In addition, the spatial distribution of the cores indicates periodic grouping of the cores into groups of $\sim 30000 \mathrm{AU}$ in size, separated by $\sim 50000 \mathrm{AU}$. The groups coincide with dust column density peaks detected by Herschel. These results show hierarchical, two-mode fragmentation in which the maternal filament periodically fragments into groups of dense cores. Critically, our results indicate that the fragmentation models for lower line-mass filaments $\left(\sim 16 M_{\odot} \mathrm{pc}^{-1}\right)$ fail to capture the observed properties of the ISF. We also find that the protostars identified with Spitzer and Herschel in the ISF are grouped at separations below $\sim 17000 \mathrm{AU}$. In contrast, young stars with disks do not show significant grouping. This suggests that the grouping of dense cores is partially retained over the protostar lifetime, but not over the lifetime of stars with disks. This is in agreement with a scenario where protostars are ejected from the maternal filament by the slingshot mechanism, a model recently proposed for the ISF. The separation distributions of the dense cores and protostars may also provide an evolutionary tracer of filament fragmentation.
\end{abstract}

Key words. ISM: clouds - ISM: structure - stars: formation - ISM: individual objects: OMC-2 - radio continuum: ISM

\section{Introduction}

Filamentary structures are fundamental building blocks of the molecular clouds of the interstellar medium (ISM), manifesting themselves over wide ranges of sizes $(\sim 0.1-100 \mathrm{pc})$, masses $\left(\sim 1-10^{5} M_{\odot}\right)$, and line-masses $\left(\lesssim 1000 M_{\odot} \mathrm{pc}^{-1}\right)$ (e.g., Bally et al. 1987; Hacar et al. 2013; Alves de Oliveira et al. 2014; Kainulainen et al. 2013, 2016; Abreu-Vicente et al. 2016). Specifically, filaments that have line-masses greatly in excess to the critical value of the self-gravitating, thermally supported, non-magnetised, infinitely long equilibrium model, i.e., $\gg 16 M_{\odot} \mathrm{pc}^{-1}$ (Ostriker 1964), contain large enough mass reservoirs to give birth to high-mass stars and star clusters (e.g., Pillai et al. 2006; Beuther et al. 2010, 2015a; Henning et al. 2010; Schneider et al. 2012; Kainulainen et al. 2013; Stutz \& Gould 2016; Contreras et al. 2016). This makes understanding their fragmentation and gravitational collapse important for Galactic-scale star formation. We refer to these

\footnotetext{
* The reduced continuum image (FITS file) is only available at the CDS via anonymous ftp to cdsarc.u-strasbg. fr (130.79.128.5) or via

http://cdsarc.u-strasbg.fr/viz-bin/qcat?J/A+A/600/A141
}

filaments as highly super-critical filaments in this paper. The physics governing the evolution of the highly super-critical filaments may be radically different from those of near-critical filaments, especially due to their strong global gravitational potential that may crucially affect their evolution (Stutz \& Gould 2016). Therefore, the fragmentation properties of highly supercritical filaments should not be extrapolated from studies of nearcritical filaments, but dedicated studies are necessary.

The main obstacle in studying the fragmentation of highly super-critical filaments is the observational challenge. They show fragmentation down to (at least) 1500 AU scales (e.g., Takahashi et al. 2013; Teixeira et al. 2016) and can be several parsecs long. This means that building a complete view of their structure requires high resolution mapping over a large area. Coupling the gas structure with star formation also requires an accurate census of the young (proto-) stellar population of the cloud; this limits the possible targets to distances closer than $\sim 1 \mathrm{kpc}$ where such a census can be attained (e.g., Evans et al. 2009; Gutermuth et al. 2011; Megeath et al. 2012; Stutz et al. 2013; Megeath et al. 2016).

Currently, the above observational challenges limit the number of possible targets to exactly one: the "integral-shaped 
filament" (ISF; e.g., Bally et al. 1987; Peterson \& Megeath 2008) in the Orion A molecular cloud. At the distance of $420 \mathrm{pc}$ (e.g., Schlafly et al. 2014), the ISF is the nearest highly supercritical filament, and especially the nearest such filament in a giant molecular cloud that exhibits high-mass star formation. The ISF, located in the northern part of Orion $\mathrm{A}$, is an $\sim 8 \mathrm{pc}$ long structure with the line-mass of $385 \times(d / p c)^{3 / 8} M_{\odot} \mathrm{pc}^{-1}$ from $0.05 \mathrm{pc}<d<8.5 \mathrm{pc}$ of projected separation from the filament ridge (Stutz \& Gould 2016). Importantly, the young stellar population of Orion A is well characterised with Spitzer (Megeath et al. 2012) and Herschel (the Herschel Orion Protostar Survey, HOPS; Furlan et al. 2016). Combined, these properties make the ISF an outstanding, and currently unique, region in which to study the physics of how highly super-critical filamentary gas fragments into (high- and low-mass) protostars. While several other highly super-critical filaments are known (e.g., Jackson et al. 2010; Hill et al. 2011; Kainulainen et al. 2013; Abreu-Vicente et al. 2016; Henshaw et al. 2016), their larger distances do not allow an analysis as detailed as can be performed in the ISF.

While the Orion A cloud has been a target of a myriad of studies (see Fig. 1 in Meingast et al. 2016), the sensitivity and resolution of the interferometer arrays have only recently enabled detailed fragmentation studies of the ISF. Teixeira et al (2016) and Takahashi et al. (2013) have analysed fragmentation in sub-parsec-sized sections of the ISF in $\sim 1600$ AU resolution (for studies in lower spatial resolution, see, e.g., Bally et al 1987; Johnstone \& Bally 1999; Chini et al. 1997; Stutz \& Gould 2016). These observations have established evidence of quasiperiodic fragment separations and hierarchical fragmentation in the filament. However, the relatively small areas covered by the surveys have hampered the statistical analyses of their fragment distributions. Similarly, no work so far has directly linked the distribution statistics of fragments to those of the young stellar population in the ISF.

We report in this paper the most sensitive fragmentation study of a highly super-critical filament to date, based on our ALMA interferometer study of the ISF and the census of the young stellar population derived by HOPS. Our mapping more than doubles the area of the ISF studied previously in a similar resolution, also reaching higher sensitivity. Our specific goals in this paper are to determine the fragmentation length scales of the filament, to look for preferential fragmentation scales and grouping of fragments, and to establish the relationship of the fragmentation scales to the distribution of the young stellar population in the ISF.

\section{Observations and methods}

\subsection{ALMA $3 \mathrm{~mm}$ continuum observations}

We used the ALMA interferometer during Cycle 2 to observe the northern part of the ISF at $3 \mathrm{~mm}$ continuum emission and $\mathrm{C}^{18} \mathrm{O}$ line emission (ESO Project ID 2013.1.01114.S, PI Kainulainen). In this paper we only use the continuum emission data. Both the 12-m and the 7-m (Atacama Compact Array; ACA) arrays were employed. The 7-m array observations were conducted on 16th August 2014 and the 12-m array observations in a compact configuration on 16th January 2015. We covered a region $\sim 3 \times 11^{\prime}$ $(\sim 0.37 \times 1.34 \mathrm{pc})$ in size in OMC-2 and OMC-3 with mosaicing observations. The footprints of the $12-\mathrm{m}$ and $7-\mathrm{m}$ arrays are shown in Fig. 1. During the observations, three of the four correlator sidebands, each $2 \mathrm{GHz}$ wide, were set to central frequencies of $107.29217 \mathrm{GHz}, 96.97848 \mathrm{GHz}$, and $98.97848 \mathrm{GHz}$.
Table 1. Parameters of the ALMA observations.

\begin{tabular}{|c|c|c|}
\hline Parameter & 12-m array & 7-m array \\
\hline Primary beam & $\sim 60^{\prime \prime}$ & $\sim 105^{\prime \prime}$ \\
\hline Max recoverable scale ${ }^{a}$ & $25^{\prime \prime}$ & $42^{\prime \prime}$ \\
\hline Synthesized beam & $3^{\prime \prime} .2 \times 1^{\prime \prime} .7$ & $19^{\prime \prime} .9 \times 10^{\prime \prime} .3$ \\
\hline Beam position angle & $71^{\circ}$ & $76^{\circ}$ \\
\hline No. of mosaic points & 127 & 42 \\
\hline On-source time & $78 \mathrm{~min}$ & 3h 44 min \\
\hline Baseline length range & $\begin{array}{l}15.1-348.5 \mathrm{~m} \\
5.0-116.2 \mathrm{k} \lambda\end{array}$ & $\begin{array}{c}8-48.9 \mathrm{~m} \\
2.7-16.3 \mathrm{k} \lambda\end{array}$ \\
\hline Achieved rms & $0.14 \mathrm{mJy}$ beam $^{-1}$ & $2.3 \mathrm{mJy} \mathrm{beam}^{-1}$ \\
\hline
\end{tabular}

Notes. ${ }^{(a)}$ According to the ALMA Cycle 2 Proposer's Guide.

The fourth sideband was set to the frequency of the $\mathrm{C}^{18} \mathrm{O}$ line at $109.749042 \mathrm{GHz}$. The relevant observational parameters are listed in Table 1.

During the 12-m array observations, J0423-0120 and Uranus were observed in the beginning of the measurement set for bandbass and flux calibration, respectively. J0517-0520 was observed every $\sim 10$ min during the science observations for phase calibration. A similar sequence was employed during the 7-m array observations, using J0607-0834 for bandbass calibration, Uranus or J0423-013 for flux and amplitude calibration, and J0541-0541 for phase calibration.

Both the 7-m and 12-m array data were calibrated and imaged using the casa version 4.5.0. The 12-m array data were calibrated using the ALMA calibration pipeline (part of casa 4.5.0). The 7-m data were calibrated using the calibration scripts prepared by the ALMA Regional Center experts; these are included in the standard data delivery and they available in the archive with the data.

We imaged the 7-m and 12-m array data both separately and simultaneously using the clean task of casa. Prior to cleaning, the relative weights of the 7-m and 12-m array visibility data were adjusted with the casa task statwt. We excluded from the cleaning the channels at the edges of the bands and the channels coinciding with prominent emission lines. The total bandwidth used in the imaging was $11.1 \mathrm{GHz}$ (including two polarizations).

During cleaning, we used the "natural" weighting scheme. We first ran the clean task without any masking of emission; the resulting image of this first cleaning was used to make the cleaning mask; this was done by setting boxes around significant emission structures in the cleaned map. The cleaning was then repeated with this mask, yielding the final, cleaned map. This procedure was repeated for 7-m array and 12-m array separately, and also for the two arrays together. The final maps are shown in Fig. 3. The final synthesised beam size full width half max, FWHM) of the combined 7-m and 12-m array map is 3 '.75 $\times 2$ '.27 $(\sim 1600 \mathrm{AU} \times 950 \mathrm{AU})$ and the beam position angle $71^{\circ}$. The root-mean-square (rms) noise measured from an approximately emission free area is $\sigma_{\text {rms }}=0.23 \mathrm{mJy}$ beam $^{-1}$. We use this combined map in the remainder of this paper and refer to it as the ALMA map.

We further investigate the sensitivity of the ALMA map by inspecting the amplitudes of its Fourier modes. Consider the ALMA map, $f(x, y)$, its dimensions, $S_{x}$ and $S_{y}$, and its discrete Fourier transform, $F(n, m)$. The transformation between the angular scale, $\Theta\left[{ }^{\prime \prime}\right]$, and the Fourier modes is then

$\Theta=\left(\left(\frac{n}{S_{x}}\right)^{2}+\left(\frac{m}{S_{y}}\right)^{2}\right)^{-1 / 2}$. 

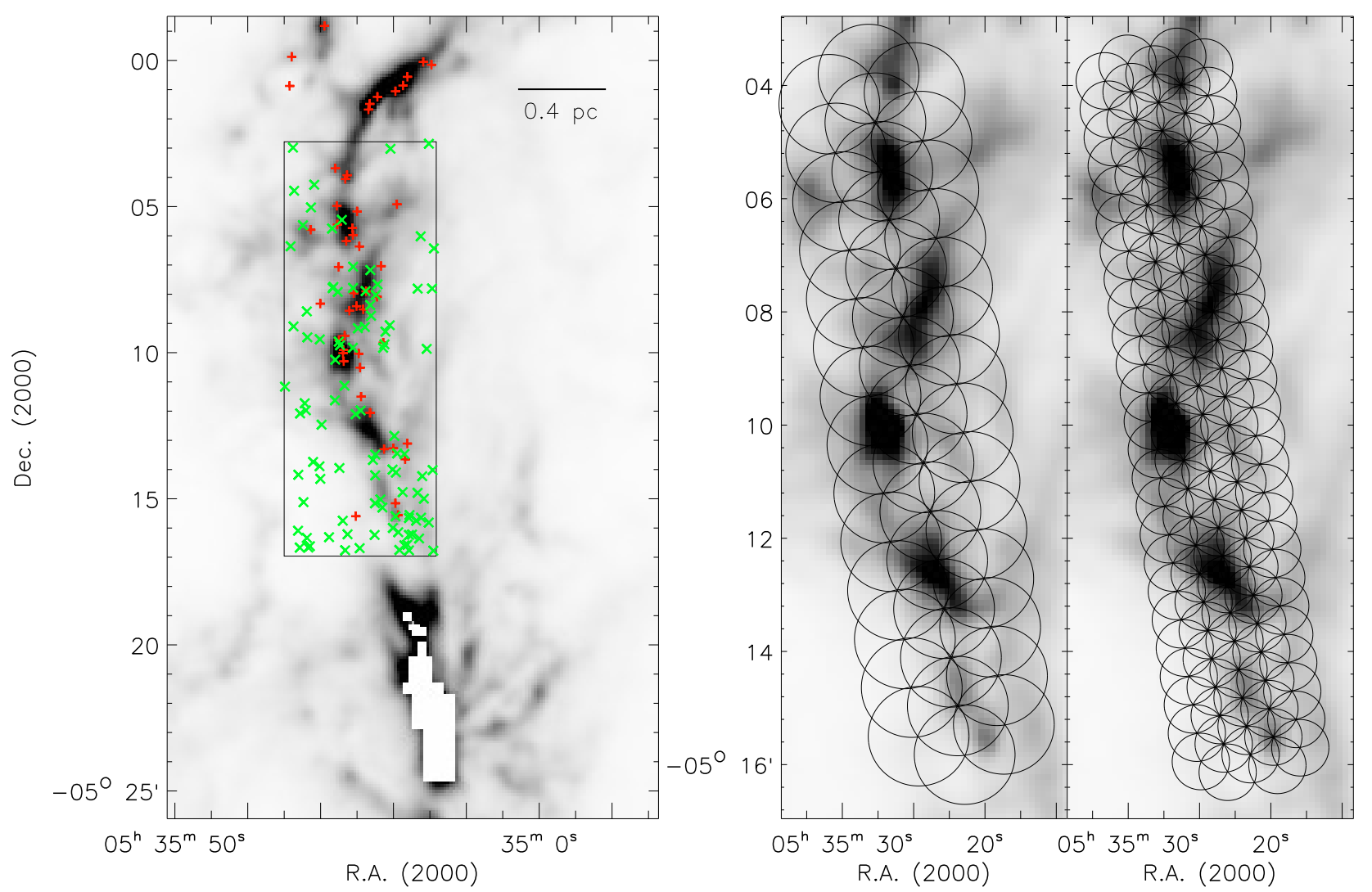

Fig. 1. Left: column density map of the northern part of the ISF, derived from Herschel data (Stutz \& Kainulainen 2015). The rectangle indicates the location of the zoom-ins shown in the right panel. The red plusses show the locations of protostars and green crosses the locations of stars with disks (Megeath et al. 2012; Furlan et al. 2016). For visibility, only disks within the squared area are shown. The white pixels result from saturation of the detector. Right: zoom-in of the left panel showing the 7-m array and 12-m array mosaic footprints.

Figure 2 shows the amplitudes of the Fourier modes as a function of the angular scale. The figure also shows the mean and standard deviation of the amplitudes, calculated within a $0.5^{\prime \prime}$ wide window. To improve visibility, only the amplitudes at scales larger than $42^{\prime \prime}$ (the filtering scale of the 7-m array) are plotted; at smaller scales the density of points is shown instead. The figure demonstrates the quality of the data: between $\sim 5-30^{\prime \prime}$, the relationship is smooth and power-law-like. Below about $5^{\prime \prime}$ the relationship steepens, evidently due to beam-size effects. Above $42^{\prime \prime}$, the relationship is not well-behaved, which indicates severe filtering effects. These effects manifest themselves in the image plane, e.g., as negative bowls (e.g., surrounding the FIR3-5 complex).

We also used the Fourier amplitudes to empirically estimate the rms noise of the ALMA map as a function of the spatial scale. We measured the rms of the Fourier spectrum as a function of the spatial scale, $\sigma_{\mathrm{rms}, \mathrm{F}}(\Theta)$, within a window that has the width of $\Theta$. Note that the choice of the window size is somewhat arbitrary; the resulting rms should be considered a rough estimate. Then, an estimate of the image rms is $1 / N \times \sigma_{\mathrm{rms}, \mathrm{F}}(\Theta)$, where $N$ is the number of data points, i.e., of Fourier modes (results from Parseval's theorem). The scale-dependent rms, $1 / N \times \sigma_{\text {rms,F }}(\Theta)$, is shown in Fig. 2, lower panel. We discuss this relationship further in the context of structure identification later in the paper.

We also employ in this work the column density map of the Orion A molecular cloud derived from Herschel far-infrared emission observations by Stutz \& Kainulainen (2015). The section of the map covering the ISF is shown in Fig. 1. The map has the spatial resolution of $F W H M=18^{\prime \prime}$ (7600 AU). We refer to Stutz \& Kainulainen (2015) for the details of the map.
Finally, we also use the catalogue of Spitzer-identified protostars and stars with disks from the HOPS survey (Megeath et al. 2012 for the disks and Furlan et al. 2016 for the protostars). The protostars and stars with disks are shown in Fig. 1.

\subsection{Identification of dense cores}

To study the spatial distribution of dense cores in the ALMA map, we first need to identify such objects. Generally, identifying "cores" from column density maps is an ill-defined problem, because there is no common standard for what exactly constitutes "a core" (see discussion in, e.g., di Francesco et al. 2007; Bergin \& Tafalla 2007; André et al. 2014). Consequently, different approaches undoubtedly result in somewhat different results (e.g., Smith et al. 2008; Kainulainen et al. 2009; Beaumont et al. 2013). Here, our emphasis is in taking the advantage of the high resolution of the ALMA data, and hence, in identifying structures that are centrally concentrated at scales a few times the beamsize (i.e., $\$ 3000-4000$ AU). The ALMA data recover structures also at larger spatial scales, up to $\sim 20000 \mathrm{AU}$, due to the inclusion of the ACA array (see Fig. 2). Since the ISF shows significant extended structures (e.g., Fig. 1), this results in a varying background for the compact structures we wish to identify. Also, some regions of the map clearly contain high surface densities of local maxima. These properties are suited to a technique based on 2-dimensional model fitting (as opposed to segmentation). Finally, we also wish to adopt a well-documented and publicly available algorithm that will be straightforward to use in future studies. 

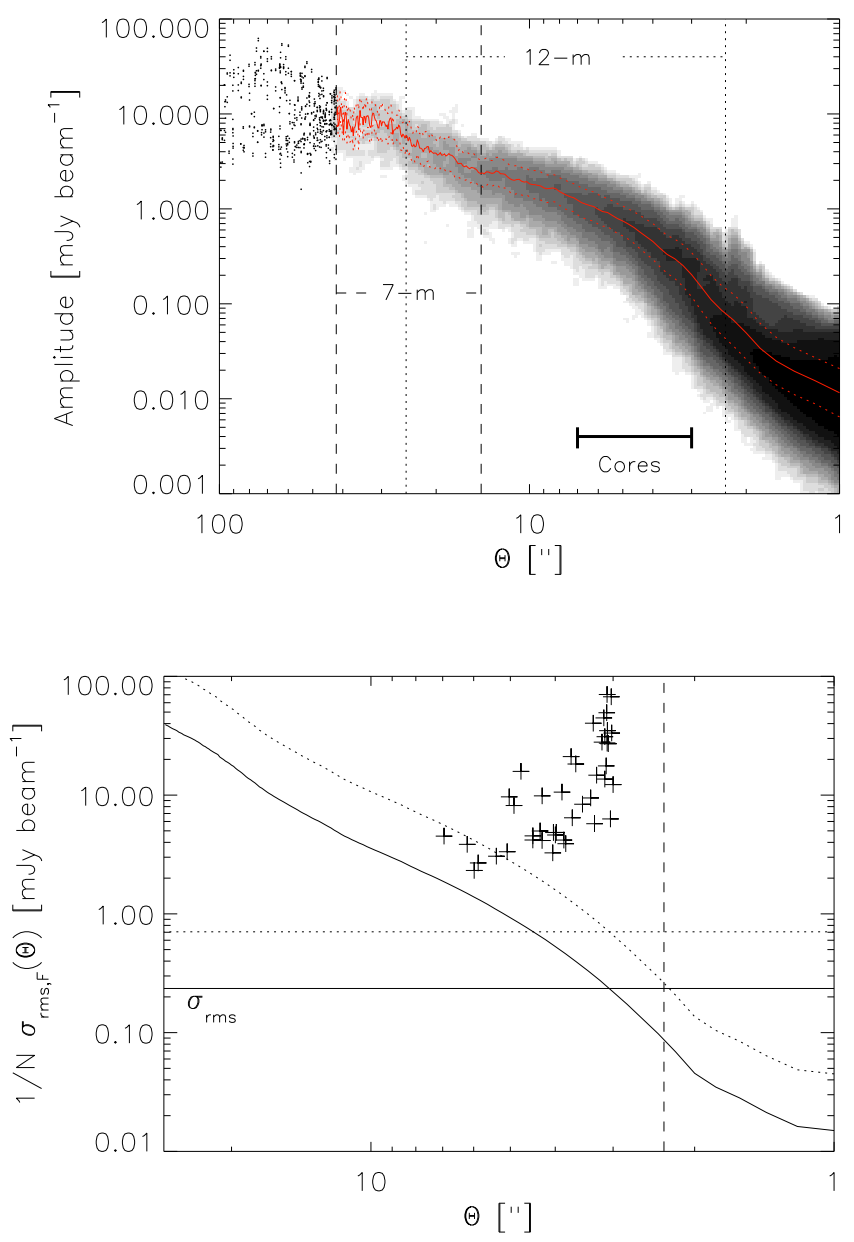

Fig. 2. Top: amplitudes of the Fourier modes of the ALMA map. The spatial scales probed by the $12-\mathrm{m}$ and $7-\mathrm{m}$ arrays are indicated with vertical lines. The size range of the identified cores is shown with a horizontal line. The mean spectrum is shown with a solid red line and the standard deviation with dotted red lines. For visibility, only data points above $25^{\prime \prime}$ are shown; at smaller scales the grey scale shows the density of data points. Bottom: rms noise measured in the Fourier domain, i.e., $1 / N \sigma_{\text {rms, } \mathrm{F}}(\Theta)$. The dotted line shows the $3 \sigma_{\text {rms,F }}(\Theta)$ value. The horizontal lines show one (solid line) and three times (dotted line) the standard deviation measured from an apparently empty region of the ALMA map, i.e., $\sigma_{\text {rms }}$. The dashed vertical line indicates the beam size. The plusses indicate the 43 compact objects identified from the ALMA map; the $y$-position is determined by the peak flux and the $x$-position by the geometric radius of the objects.

With the above considerations, we chose to use the implementation of gaussclumps in the starlink/cupid package version $2.0^{1}$. gaussclumps identifies cores by fitting 2-dimensional Gaussians at the locations defined by the highest pixel values in the map. Prior to fitting, a background is subtracted from the map using a median filter. After the peak identification and the fitting of the Gaussian profile, the profile is subtracted from the map and the residual map is used to search for the next object. The most important parameters of the algorithm are the threshold (GaussClumps.Thresh in cupid) defining the minimum acceptable value for the peak of the fitted Gaussian function and the width of the filtering function that is used to

1 Available at http://starlink.eao.hawaii.edu/starlink/ CUPID (Berry et al. 2007). The gaussclumps was originally developed by Stutzki \& Guesten (1990); see the cupid manual for the minute differences between the cupid implementation and the original version. subtract the background. We chose to use the threshold three times the rms noise calculated from an emission free region in the ALMA+ACA map, i.e., $0.23 \mathrm{mJy}$ beam $^{-1}$. This value is about two times the rms noise measured empirically from the Fourier spectrum at the size of the beam (Fig. 2). Note that not all structures above the threshold are considered as cores; if the structure profile is not well fitted by a Gaussian, the core is rejected. The filter width for the background subtraction was chosen to be $10^{\prime \prime}$, keeping in mind that we here want to concentrate on detection of objects at scales smaller than a few times the beamsize $(\$ 4000 \mathrm{AU})$. This restricts the reasonable choices of the background to roughly $9^{\prime \prime}-15^{\prime \prime}$ (3-5 times the beamsize). We experimented with different filter widths and found that between $7.5^{\prime \prime}-15^{\prime \prime}$ the choice of the width does not change the conclusions based on the two-point correlation function (see Appendix A).

Running gaussclumps resulted in detection of 43 compact objects from the ALMA map. Figure 2 (bottom panel) shows the peak fluxes and mean sizes of the objects. The weakest objects have peak fluxes about $10 \sigma_{\text {rms }}$, indicating robust detections. Most cores are also above the $3 \sigma_{\text {rms, } \mathrm{F}}(\Theta)$ level; from the five cores below $3 \sigma_{\text {rms, } \mathrm{F}}(\Theta)(\# 23,28,29,33$, and 35), all except \#23 are local emission maxima. The 43 identified objects are listed in Table 2 together with their intensities, integrated fluxes, and sizes (shown in Fig. 4).

We cross-correlated the positions of the cores with the protostars (Furlan et al. 2016) and stars with disks (Megeath et al. 2012). The objects were considered coincident if their centroids were within a $2^{\prime \prime}$ radius of the protostars or disks. 26 of the objects coincide with a protostar and four objects with a disk source (\#24 that has the identifier HOPS64, and objects \#8, \#34, and \#42, which have no HOPS identifier). These four objects all show extended emission surrounding them. It is possible that they indeed are disks, aligning by chance with emission structures along the line-of-sight. However, it is also possible that they are misclassified protostars. We performed a simple estimation of the probability of chance alignment. Consider the probability of chance alignments of $N_{\text {object }}$ circular objects and $N_{\text {disk }}$ point-like disk sources. The probability of having $N$ superpositions is

$P(N)=\frac{N_{\text {disks }} !}{N !\left(N_{\text {disks }}-N\right) !} p^{N}(1-p)^{N_{\text {disks }}-N}$,

where $p$ is the probability of one superposition, i.e., $N_{\text {cores }} \pi R_{\text {object }}^{2} / A$, where $R_{\text {object }}$ is the radius of the object and $A$ is the total considered area. Using the values $N_{\text {cores }}=43$, $N_{\text {disks }}=51, R_{\text {object }}=2^{\prime \prime}$, and $A=1.5 \times 10^{5} \square^{\prime \prime}$ results in $P(0)=83 \%$ and the expected value of $N$ of 0.2 . Against this expectation, we reclassify the four disks that coincide with ALMAidentified compact objects as protostars and consider them as likely misclassifications in Megeath et al. (2012). In particular, HOPS 64 (\#24) shows a rising SED between $1.6 \mu \mathrm{m}$ and $24 \mu \mathrm{m}$ and has been shown to have an outflow cavity in HST $1.6 \mu \mathrm{m}$ imaging (J. Booker, priv. comm.). The other sources have flat to Class II SEDs between 1.6-24 $\mu \mathrm{m}$; these may be protostars observed near a face-on inclination and/or with lower mass envelope (Furlan et al. 2016).

We refer to the 43 ALMA-identified compact objects as dense cores in the remainder of this paper. 17 of the dense cores do not coincide with protostars and are considered to be starless cores. Thus, our dense core catalogue consists of 26 protostellar cores and 17 starless cores. We note that we do not assess whether the starless cores are gravitationally bound or not (gravitationally bound cores that do not coincide with protostars are 


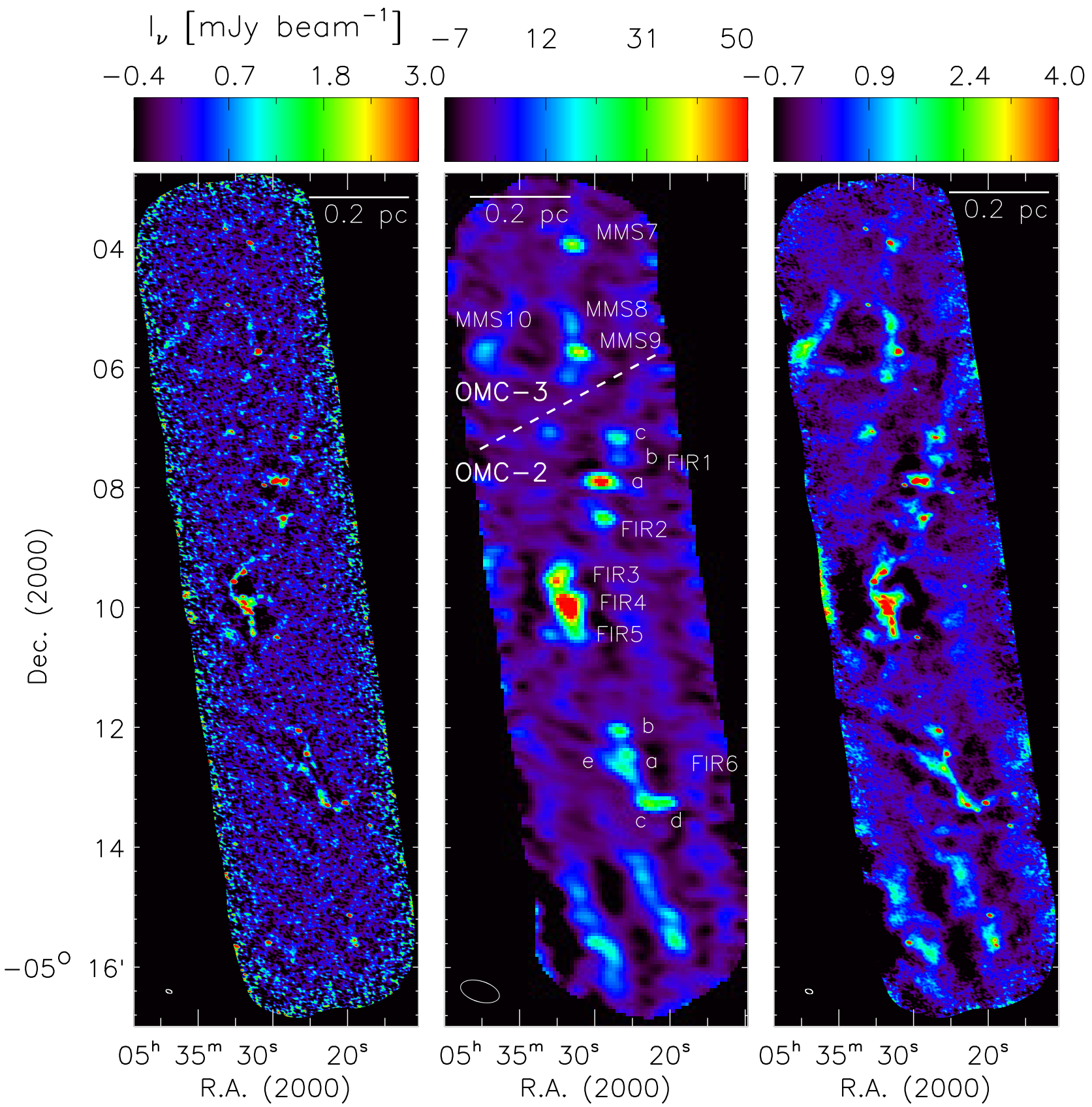

Fig. 3. 3 mm continuum emission maps of the OMC-2/3 region, observed with ALMA. The FWHM beam is shown in the lower left corner of the frames. Left: map observed with the $12-\mathrm{m}$ array. The beamsize is $3^{\prime \prime} .2 \times 1^{\prime \prime} \cdot 7$. Centre: map observed with the 7 -m array. The beamsize is $19^{\prime \prime} \cdot 9 \times 10^{\prime \prime} 3$. The region designations from Mezger et al. (1990) and Chini et al. (1997) are shown in the frame. Right: map combining the 7-m and 12-m array data. The beamsize is 3 '.75 $\times 2$ '!27. See Fig. 4 for higher resolution zoom-ins of the map.

commonly referred to as "prestellar cores"), but rather consider all starless cores together.

We note two caveats arising from the structure identification technique. Because of the background filtering of gaussclumps, we may be insensitive to low-contrast cores that have sizes of $10^{\prime \prime}$ (4000 AU) or larger. Starless cores typically have low density contrasts (e.g., Alves et al. 2001; Kandori et al. 2005; Kainulainen et al. 2007). This may affect the distribution statistics of starless cores analysed later in the paper. Protostellar cores are centrally concentrated and are unlikely to suffer from the incompleteness due to the background filter. In the future, combination of the ALMA data with single-dish measurement can be used to address this caveat. We also do not assess in this work whether the column density peaks we identify correspond to peaks in local volume density. It is possible that some identified objects are column density peaks resulting from, e.g., overlap of unrelated structures along the line of sight. Further molecular line studies should be able to address this caveat in the future.

We give rough estimates for the masses of the ALMAdetected cores using the equation

$M=\frac{F_{v} d^{2}}{B_{v}(T) \kappa_{v}}$,

where $F_{v}$ is the total integrated flux, $d$ is the distance, $B_{v}(T)$ is the Planck function at the temperature of $T$, and $\kappa_{v}$ is the mass absorption coefficient. For the temperature, we adopt $20 \mathrm{~K}$ 
(see Li et al. 2013 for gas temperature measurements from $\mathrm{NH}_{3}$ ). We calculate the mass absorption coefficient from the expression $\kappa_{v}=0.1(v / 1000 \mathrm{GHz})^{\beta} \mathrm{cm}^{2} \mathrm{~g}^{-1}$, where $\beta$ is taken to be 1.5 (e.g., Sadavoy et al. 2016). This expression assumes the gas-todust ratio of 100 . The resulting mass absorption coefficient is $\kappa_{100 \mathrm{GHz}}=0.0032 \mathrm{~cm}^{2} \mathrm{~g}^{-1}$. The masses of the cores span $0.2-$ $2.6 M_{\odot}$ (Table 2).

\section{Results}

\subsection{Structure of the OMC-2/3 at $1000 \mathrm{AU}$ resolution}

The ALMA map reveals intricate details within the OMC-2/3 region (Fig. 4). Most structures identified in the previous studies at $\sim 10^{\prime \prime}$ resolution (Mezger et al. 1990; Chini et al. 1997; Johnstone \& Bally 1999), and in the Herschel data at $\sim 18^{\prime \prime}$ resolution (Fig. 1; Stutz \& Kainulainen 2015) are resolved into multiple sub-structures. Some of the sub-structures are filamentary. In the following, we briefly describe the morphology as revealed by the ALMA map beyond the previous studies. We follow the designations of Mezger et al. (1990) and Chini et al. (1997) (shown in Fig. 3), proceeding from north to south.

MMS 7 (core \#2): the source is single peaked at the resolution of the ALMA map (as already indicated by the 4 " resolution data of Takahashi et al. 2013 at $1300 \mu \mathrm{m})$. We detect a previously undetected compact source 14" (5800 AU) north-east from MMS 7 (core \#1).

MMS 8-9 (cores \#4-7): the prominent filamentary structure detected by Mezger et al. (1990) and Chini et al. (1997) breaks into three local maxima, north- and centre-most of which correspond to MMS 8 and MMS 9, respectively.

MMS 10 (no ALMA cores): the appearance is similar to that evidenced by the Chini et al. (1997) data, with one local maximum in an elongated clump.

FIR $1 a, b, c$ (cores \#8, 10-14): the ALMA data resolves FIR 1a into four dense cores (three of them have previously been detected from arcsecond-resolution continuum emission data by Tobin et al. 2015). One of the peaks (core \#11) is close (3".5, or $\sim 1500 \mathrm{AU}$ ) to a Class 0 protostar that has exceptionally high envelope density and mass-infall rate, discovered by Stutz et al. (2013) (referred to as the PACS Bright Red Sources, PBRSs). The configuration of the two strongest peaks (cores \#11 and \#12) bears resemblance to the Bok Globule CB 244 in which a starless core and a young protostar co-exist within a common envelope (Stutz et al. 2010). FIR 1b is relatively extended in the ALMA map and does not harbour a dense core. FIR 1c contains a single compact source.

FIR 2 (cores \#15-16): two cores are found close to the peak identified by Chini et al. (1997).

FIR 3-5 (cores \#17-20, 22-30): Chini et al. (1997) identifies three sources in the region from mm-wavelength data. Midinfrared imaging at $\sim 4^{\prime \prime}$ resolution resolves eight sources in the region (Adams et al. 2012) and mm-wavelength data detect 12 fragments altogether (Shimajiri et al. 2008). The ALMA data shows how the region is inter-connected by a filamentary structure from which we identify 13 dense cores. Four cores coincide with HOPS protostars. The cores \#20 and 25 coincide with the FIR 3 and 4 (see, e.g., Furlan et al. 2014). FIR 5 does not appear as a dense core in the ALMA data, although, it is within $\sim 4$ " of the cores \#28 and 29. Previously, Shimajiri et al. (2008) suggested that the fragmentation in the area is caused by interaction with the outflow driven by FIR 3 with a nearby clump. In general, we find a rather poor correspondence between the ALMA-detected dense cores and the fragments identified by
Shimajiri et al. (2008). The ALMA data indicate that the cores are primarily arranged along a filament in this region.

FIR 6a-e (cores \#31-33, 35-38): another prominent filamentary structure in the ALMA data. The sources FIR 6a-d identified by Chini et al. (1997) are each resolved into at least two dense cores. The source FIR 6e is filamentary in the ALMA data and does not contain a compact source. Altogether, the region contains nine dense cores. The south-most tip of the structure harbours four sources within a $\sim 12000$ AU region, making it the second densest concentration of sources in the mapped area after the FIR 3-5 filament.

Our ALMA map continues about 2' south from the area mapped by Chini et al. (1997). This area contains two prominent structures elongated in north-south direction. The western of these is clearly visible in the Herschel-derived column density map (Stutz \& Kainulainen 2015, see Fig. 1). The eastern structure is not visible in the Herschel data, but is clearly visible in the $850 \mu \mathrm{m}$ emission data (Lane et al. 2016, also partly visible in the data of Johnstone \& Bally 1999) and also detected in ammonia line emission (J. Pineda, priv. comm.). The area contains four dense cores (\#40-43), listed in Table 2.

\subsection{Nearest neighbour separations of the dense cores, protostars, and disks}

Our goal is to characterise the fragmentation in the ISF down to $\sim 1000$ AU scales with a special emphasis in searching for preferential fragmentation scales, and to subsequently compare the characteristics to those of the young stellar population of the filament. First, we compute the basic statistics of the projected nearest neighbour separations of the objects in the ALMA-covered region. Note that the sample of 43 dense cores consists of 17 starless cores and 26 protostellar cores. However, there are altogether 37 protostars in the mapped area (33 from Furlan et al. 2016, and four re-classified by us form Megeath et al. 2012); not all protostars coincide with an object identified as a core, which may indicate that some HOPS protostars may be misclassified stars with disks. We report the mean and median nearest neighbour separation, $d_{\mathrm{nn}}$, for all different object classes in Table 3 . The mean projected nearest neighbour separations of the dense cores are similar to those seen in the OMC-3 region of the ISF by Takahashi et al. (2013). They are a factor of two larger than those measured in the ONC-1n region by Teixeira et al. (2016). The mean nearest neighbour separations of protostars and stars with disks are roughly a factor of two smaller than in Orion A on average (Megeath et al. 2016). However, we show below and in Sect. 3.3 that the cores, protostars, and disks are clearly grouped in different ways. This implies that the mean nearest neighbour separations may not be very useful measures, especially when averaged over a variety of environments.

Figure 5 shows the cumulative distribution functions (CDFs) of the nearest neighbour separations of all cores, starless cores, protostars, and stars with disks. The CDFs show that the nearest neighbour distribution of starless cores and protostellar cores are different, with the starless cores exhibiting a larger fraction of short separations. The short core separations increase down to the resolution limit of the ALMA data (1200 AU). Thus, we do not detect a preferential nearest-neighbour separation for the dense cores. Note here the caveat that our starless core sample may be incomplete and may include objects that are enhancements only in column density, not in volume density (see Sect. 2.2). The CDFs also show that the distribution of the dense cores is different from that of protostars and disks, specifically so 
J. Kainulainen et al.: Resolving the fragmentation of high line-mass filaments

Table 2. 3 mm compact sources identified from the ALMA data.

\begin{tabular}{|c|c|c|c|c|c|c|c|c|}
\hline$\#$ & RA (2000) & Dec (2000) & $\begin{array}{c}I_{v}(\text { peak }) \\
{\left[\mathrm{mJy} \mathrm{beam}^{-1}\right]}\end{array}$ & $\begin{array}{c}F_{v} \\
{[\mathrm{mJy}]}\end{array}$ & $\operatorname{Size}^{a}$ & $\begin{array}{c}M \\
{\left[M_{\odot}\right]}\end{array}$ & Association $^{b}$ & Class. $^{c}$ \\
\hline \multicolumn{9}{|c|}{ OMC-3/MMS 7} \\
\hline 1 & $05: 35: 28.165$ & $-05: 03: 41.25$ & 13.6 & 8.3 & $4 " .1 \times 22^{\prime \prime} 4$ & 0.4 & HOPS85 & ps(flat) \\
\hline 2 & 05:35:26.541 & $-05: 03: 54.91$ & 49.4 & 39.2 & $4 \prime .4 \times 22^{\prime \prime} 5$ & 1.9 & HOPS84 & $\operatorname{ps}(\mathrm{I})$ \\
\hline \multicolumn{9}{|c|}{ OMC-3/MMS 8-10 } \\
\hline 3 & 05:35:27.999 & $-05: 04: 57.36$ & 17.6 & 7.5 & $3 \prime \prime 9 \times 2.15$ & 0.4 & HOPS81 & $\operatorname{ps}(0)$ \\
\hline 4 & $05: 35: 25.950$ & $-05: 05: 43.24$ & 40.3 & 42.2 & $2 \prime .6 \times 44^{\prime \prime} 0$ & 2.1 & HOPS78 & $\operatorname{ps}(0)$ \\
\hline 5 & $05: 35: 26.120$ & $-05: 05: 45.79$ & 4.8 & 8.6 & $6 " .2 \times 22^{\prime \prime} 6$ & 0.4 & - & starless \\
\hline 6 & $05: 35: 26.666$ & $-05: 06: 10.60$ & 4.6 & 6.3 & $3 \prime .8 \times 2 " .4$ & 0.3 & HOPS75 & $\mathrm{ps}(0)$ \\
\hline 7 & 05:35:24.849 & $-05: 06: 21.63$ & 5.7 & 3.9 & $4 \prime \prime 3 \times 2 \prime \prime 5$ & 0.2 & HOPS74 & ps(flat) \\
\hline \multicolumn{9}{|c|}{ OMC-2/FIR 1-2 } \\
\hline 8 & $05: 35: 21.870$ & $-05: 07: 01.64$ & 8.4 & 4.6 & $4 " .8 \times 2 " \prime 6$ & 0.2 & - & $\mathrm{ps}^{d}$ \\
\hline 9 & $05: 35: 27.731$ & $-05: 07: 03.73$ & 5.0 & 12.0 & $5 \prime .4 \times 3 \prime .5$ & 0.6 & HOPS73 & $\mathrm{ps}(0)$ \\
\hline 10 & $05: 35: 23.507$ & $-05: 07: 09.79$ & 18.3 & 17.0 & $5 \prime .1 \times 2, .5$ & 0.8 & - & starless \\
\hline 11 & $05: 35: 24.188$ & $-05: 07: 52.75$ & 9.7 & 38.1 & $6 " .4 \times 4{ }^{\prime \prime} 0$ & 1.9 & - & starless \\
\hline 12 & $05: 35: 24.847$ & $-05: 07: 53.72$ & 15.8 & 52.1 & $6 " .3 \times 33^{\prime \prime} 0$ & 2.6 & 019003 & $\mathrm{PBRS}^{e}$ \\
\hline 13 & $05: 35: 25.584$ & $-05: 07: 57.34$ & 33.3 & 15.2 & $3 \prime \prime 8 \times 2 " .4$ & 0.7 & HOPS71 & ps(I) \\
\hline 14 & $05: 35: 22.502$ & $-05: 08: 04.60$ & 4.2 & 5.1 & $5 \prime \prime 9 \times 2.5$ & 0.3 & HOPS70 & ps(flat) \\
\hline 15 & 05:35:24.009 & $-05: 08: 27.65$ & 4.2 & 8.0 & $6 " .4 \times 3 " .2$ & 0.4 & - & starless \\
\hline 16 & $05: 35: 24.287$ & $-05: 08: 30.65$ & 21.1 & 33.6 & $4 \prime \prime 2 \times 3 " ! 2$ & 1.7 & HOPS68 & $\mathrm{ps}(\mathrm{I})$ \\
\hline \multicolumn{9}{|c|}{ OMC-2/FIR 3-5 } \\
\hline 17 & $05: 35: 26.928$ & $-05: 09: 24.40$ & 8.2 & 30.4 & $5 \prime \prime 7 \times 4^{\prime \prime} .2$ & 1.5 & HOPS66, SOF1 & ps(flat) \\
\hline 18 & $05: 35: 27.197$ & $-05: 09: 26.10$ & 3.3 & 5.6 & $3 \prime \prime 7 \times 4 " .4$ & 0.3 & - & starless \\
\hline 19 & $05: 35: 27.512$ & $-05: 09: 29.54$ & 3.3 & 13.2 & $4^{\prime \prime} 0 \times 66^{\prime \prime} 6$ & 0.7 & - & starless \\
\hline 20 & $05: 35: 27.618$ & $-05: 09: 34.06$ & 70.3 & 51.7 & $3 \prime \prime 7 \times 22^{\prime \prime} 6$ & 2.5 & $\begin{array}{l}\text { HOPS } 370, \\
\text { FIR3, SOF2N, } \\
\text { CO outflow }\end{array}$ & ps(I) \\
\hline 21 & $05: 35: 21.566$ & $-05: 09: 38.62$ & 12.3 & 4.7 & $3 \prime .3 \times 2.77$ & 0.2 & HOPS65 & $\mathrm{ps}(\mathrm{I})$ \\
\hline 22 & $05: 35: 26.992$ & $-05: 09: 48.62$ & 4.9 & 7.3 & $5 \prime 3 \times 2.9$ & 0.4 & - & starless \\
\hline 23 & $05: 35: 27.528$ & $-05: 09: 50.64$ & 2.3 & 14.0 & $9 \prime .2 \times 3 \prime \prime 9$ & 0.7 & - & starless \\
\hline 24 & $05: 35: 26.928$ & $-05: 09: 54.51$ & 6.4 & 10.1 & $2 " .8 \times 4 " .6$ & 0.5 & HOPS64 & $\mathrm{ps}^{d}$ \\
\hline 25 & $05: 35: 27.015$ & $-05: 09: 59.59$ & 3.9 & 5.9 & $5 \prime .9 \times 2 \prime .5$ & 0.3 & $\begin{array}{l}\text { HOPS108, } \\
\text { FIR4, SOF3 }\end{array}$ & $\mathrm{ps}(0)$ \\
\hline 26 & $05: 35: 26.797$ & $-05: 10: 04.81$ & 4.5 & 11.6 & $3 " .6 \times 5 " .6$ & 0.6 & - & starless \\
\hline 27 & $05: 35: 26.455$ & $-05: 10: 05.10$ & 10.6 & 20.6 & $2 \prime \prime 8 \times 5 \prime \prime 3$ & 1.0 & - & starless \\
\hline 28 & $05: 35: 26.396$ & $-05: 10: 15.20$ & 4.5 & 37.3 & $5 \prime \prime 1 \times 9 \prime \prime 4$ & 1.8 & - & starless \\
\hline 29 & $05: 35: 26.325$ & $-05: 10: 25.13$ & 3.8 & 24.7 & $5 \prime \prime 0 \times 7 " .6$ & 1.2 & - & starless \\
\hline 30 & 05:35:24.717 & $-05: 10: 29.78$ & 27.4 & 15.2 & $3 \prime \prime 9 \times 2 \prime .4$ & 0.7 & HOPS368, SOF5 & $\mathrm{ps}(\mathrm{I})$ \\
\hline \multicolumn{9}{|c|}{ OMC-2/FIR 6} \\
\hline 31 & $05: 35: 23.278$ & $-05: 12: 03.15$ & 44.7 & 34.3 & $2 " \prime 4 \times 4 " .0$ & 1.7 & $\begin{array}{l}\text { HOPS60, FIR6b, } \\
\text { CO outflow }\end{array}$ & $\mathrm{ps}(0)$ \\
\hline 32 & $05: 35: 22.745$ & $-05: 12: 26.47$ & 9.9 & 24.9 & $4 \prime .0 \times 4 " .5$ & 1.2 & FIR6a & starless \\
\hline 33 & $05: 35: 22.939$ & $-05: 12: 40.70$ & 2.7 & 14.8 & $7 " .2 \times 4 " .8$ & 0.7 & FIR6a & starless \\
\hline 34 & $05: 35: 18.221$ & $-05: 13: 05.83$ & 14.7 & 7.1 & $4 " .1 \times 2 " .6$ & 0.4 & - & $\mathrm{ps}^{d}$ \\
\hline 35 & $05: 35: 21.705$ & $-05: 13: 13.08$ & 3.1 & 12.7 & $4 \prime \prime 1 \times 7 ! \prime 0$ & 0.6 & FIR6c & starless \\
\hline 36 & $05: 35: 20.138$ & $-05: 13: 15.40$ & 67.3 & 40.9 & $3 \prime .8 \times 2 \prime .4$ & 2.0 & $\begin{array}{l}\text { HOPS59, FIR6d, } \\
\text { CO outflow }\end{array}$ & ps(flat) \\
\hline 37 & $05: 35: 21.348$ & $-05: 13: 17.54$ & 27.9 & 22.5 & $4^{\prime \prime} .2 \times 2 " .4$ & 1.1 & $\begin{array}{l}\text { HOPS } 409, \text { FIR6c, } \\
\text { CO outflow }\end{array}$ & $\operatorname{ps}(0)$ \\
\hline 38 & $05: 35: 20.760$ & $-05: 13: 22.51$ & 4.6 & 9.1 & $5 \prime \prime 3 \times 3 " .0$ & 0.4 & FIR6c & starless \\
\hline 39 & $05: 35: 18.516$ & $-05: 13: 38.20$ & 34.9 & 9.5 & $3 \prime \prime 9 \times 2 \prime .5$ & 0.5 & HOPS58 & ps(flat) \\
\hline 40 & $05: 35: 19.872$ & $-05: 15: 08.02$ & 27.1 & 16.2 & $3 \prime .9 \times 2 ! .4$ & 0.8 & HOPS57 & ps(flat) \\
\hline 41 & $05: 35: 19.503$ & $-05: 15: 32.70$ & 4.1 & 9.3 & $4 " .6 \times 3 " .9$ & 0.5 & HOPS56 & $\mathrm{ps}(0)$ \\
\hline 42 & $05: 35: 25.265$ & $-05: 15: 35.25$ & 31.0 & 17.7 & $4 " .1 \times 22^{\prime \prime} 4$ & 0.9 & - & $\mathrm{ps}^{d}$ \\
\hline 43 & $05: 35: 19.413$ & $-05: 15: 37.98$ & 9.5 & 11.0 & $2 " .6 \times 4 " .3$ & 0.5 & - & starless \\
\hline
\end{tabular}

Notes. ${ }^{(a)}$ The FWHM sizes of the object. ${ }^{(b)}$ Association with the far-infrared sources as labelled in (Mezger et al. 1990; Chini et al. 1997), HOPS protostars (Furlan et al. 2016), PBRSs (Stutz et al. 2013), SOFIA mid-infrared detections (Adams et al. 2012), and CO outflows (Shimajiri et al. 2008, 2009). ${ }^{(c)}$ Evolutionary classification of the object. Classification of protostars (ps) is according to Furlan et al. (2016) unless otherwise stated. The objects without identified protostars are considered starless. ${ }^{(d)}$ The core coincides with a disk source (Megeath et al. 2012). Due to the association with extended $3 \mathrm{~mm}$ emission and the low expected number of chance superpositions, we re-classify the source as a protostar. (e) PBRS, i.e., a Class 0 source with a very red spectral energy distribution (Stutz et al. 2013). 

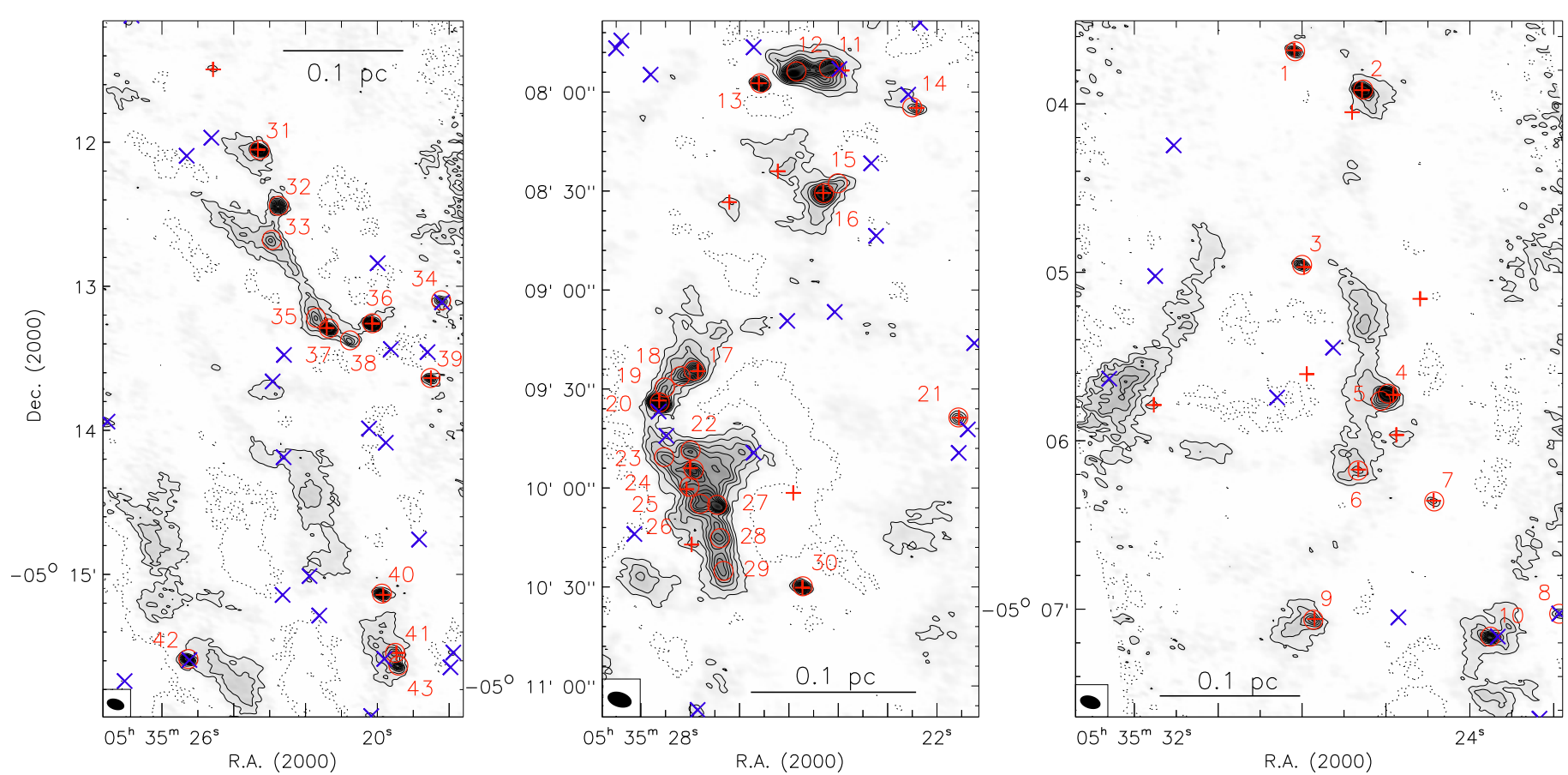

Fig. 4. ALMA $3 \mathrm{~mm}$ continuum emission maps of the OMC-2/3 region (combined $12-\mathrm{m}$ and $7-\mathrm{m}$ array data). The beamsize is 3 '.75 $722^{\prime \prime 2} 27$ and the FWHM beam is shown in the lower left corner of the frames. The contours are drawn in $3 \sigma_{\text {rms }}$ intervals, with $\sigma_{\text {rms }}=0.23 \mathrm{mJy} \mathrm{beam}^{-1}$. The dotted contour is at $-3 \sigma_{\text {rms }}$. The red circles and numbers indicate the objects detected by gaussclumps (listed in Table 2 ). These objects are regarded as dense cores, unless they coincide with a star with disk. The red plusses indicate protostars and the blue crosses stars with disks.

Table 3. Properties of the nearest neighbour distributions.

\begin{tabular}{lcccc}
\hline \hline Objects & No. & $\begin{array}{c}\left\langle d_{\mathrm{nn}}\right\rangle \\
{[\mathrm{kAU}]}\end{array}$ & $\begin{array}{c}\text { Median } d_{\mathrm{nn}} \\
{[\mathrm{kAU}]}\end{array}$ & $\begin{array}{c}\langle M\rangle \\
{\left[M_{\odot}\right]}\end{array}$ \\
\hline All dense cores & 43 & 7.7 & 4.2 & 0.9 \\
Starless cores & 17 & 11.5 & 6.1 & 0.8 \\
Protostellar cores & 26 & 12.9 & 11.7 & 1.0 \\
Protostars & 37 & 11.4 & 10.6 & - \\
Stars with disks & 51 & 8.7 & 7.1 & - \\
\hline
\end{tabular}

that cores have an excess of short separations compared to disks and protostars.

\subsection{Two-point correlation function of the dense core, protostar, and disk separations}

To analyse the grouping of the ALMA cores, we examine the two-point correlation function, $\xi(r)$, of the core separations. The function describes the excess probability per unit area, in comparison to the expectation from random placement, of an object being located at a separation $r$ from another object. We adopt the Landy \& Szalay (1993) estimator for the two-point correlation function

$\xi(r)=\frac{\frac{2}{N_{\text {cores }}\left(N_{\text {cores }}-1\right)} D D(r)-\frac{2}{N_{\text {cores }}^{2}} D R(r)+\frac{2}{N_{\text {cores }}\left(N_{\text {cores }}-1\right)} R R(r)}{\frac{2}{N_{\text {cores }}\left(N_{\text {cores }}-1\right)} R R(r)}$,

where $D D(r)$ is the observed distribution of pair separations (for $N_{\text {cores }}$ cores), $D R(r)$ is the distribution of pair separations when one core is taken from the observed sample and one from a random sample (resulting in $N_{\text {cores }}^{2}$ pairs), and $R R(r)$ is the distribution of pair separations of randomly placed cores

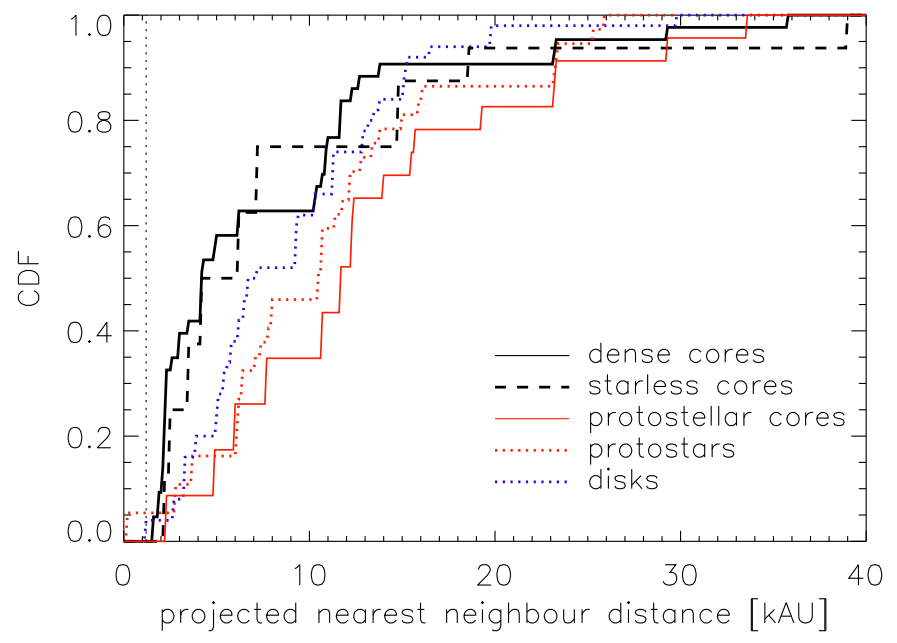

Fig. 5. Cumulative distribution function, CDF, of the projected nearest neighbour separations for all dense cores (solid black line), starless cores (dashed black line), protostellar cores (red line), protostars (dotted red line), and stars with disks (dotted blue line). The vertical dotted line shows the $1200 \mathrm{AU}$ resolution limit of the ALMA data.

(for $N_{\text {cores }}$ cores). The $\xi(r)=0$ indicates a distribution similar to random distribution; the positive and negative values indicate excess and deficit of separations, respectively. We compute the random separation distributions $(R R(r))$ with a Monte Carlo simulation, in which we place $N_{\text {cores }}=43$ points in the ALMA map area and compute their pair separation distribution. This is repeated 10000 times (and the resulting distribution is divided by 100000$)$ to obtain adequate statistics. The cross term $(D R(r))$ is computed via a similar simulation, only with one point taken from among the observed core locations and 

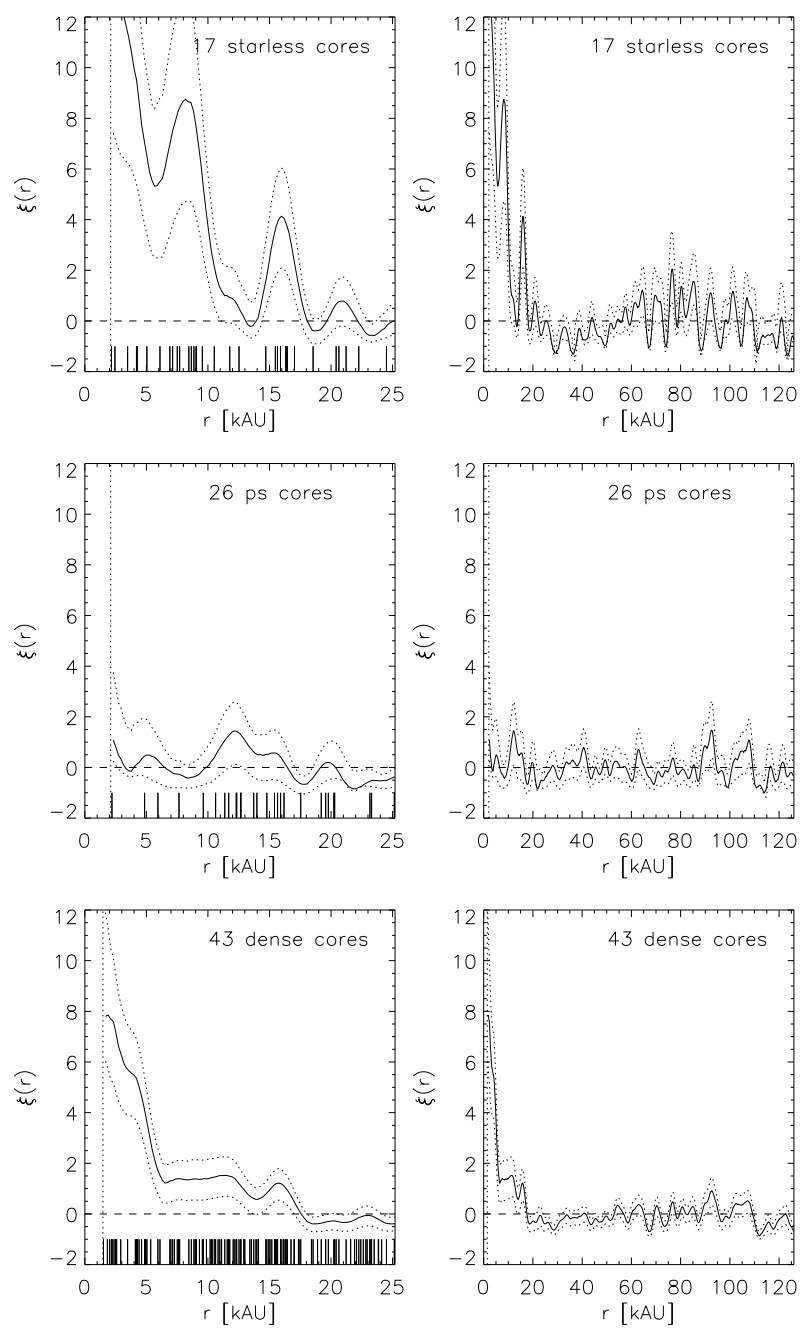

Fig. 6. Two-point correlation function of the separations of all ALMA dense cores, starless cores, and protostellar cores identified from the ALMA data. The frames on the left show the separations until $25 \mathrm{kAU}$ and the frames on the right until $125 \mathrm{kAU}$. The dotted curves show the confidence intervals given by three times the $25 \%$ and $75 \%$ quartiles. The vertical dotted line shows the minimum separation. The dashed horizontal line, drawn at zero, indicates a random distribution. The short vertical lines at the bottom of the panels show the observed separations (only in the panels on the left).

another from among random locations. We calculate all separation distributions using a Gaussian density estimator that has a $F W H M=5^{\prime \prime}(2100 \mathrm{AU})$. For example, $D D(r)$ then describes the separation distribution within 2100 AU bin centred at $r$. We tested whether the choice of the FWHM affects the main conclusions we derive using two-point correlation functions significantly, and it does not.

We compute an uncertainty estimate of the two-point correlation function using bootstrapping. We randomly draw $N_{\text {cores }}$ points from all observed core locations and calculate their separation distribution, resulting in a simulated sample and the corresponding $D D(r)$ function. The two-point correlation function is then computed following the steps described above. The procedure is repeated 10000 times, resulting in a sample of $10000 \xi(r)$ functions. For each $r$, we compute the $25 \%$ and $75 \%$ quartiles of these functions and use the quartiles as uncertainty estimates (shown in Figs. 6 and 7).
The two-point correlation functions of the 17 starless cores, 26 protostellar cores, and all 43 cores together are shown in Fig. 6. All are close to zero above 17000 AU (however, see Sect. 4). The two-point correlation function of the starless cores is dramatically different from that of the protostellar cores. The starless cores show a systematic increase of grouping at short separations down to our resolution limit. The protostellar cores are in agreement with a random distribution practically at all separations. However, one has to recognise that the number of detections especially below $\sim 10000$ AU is very small, and consequently, the uncertainty is large. The two-point correlation function of all 43 cores together is sampled reasonably well down to our resolution limit, and it shows significant grouping below $17000 \mathrm{AU}$ and strong grouping below 6000 AU. These results are not strongly affected by possible misclassification of core-like objects (see Appendix C).

We additionally analyse the two-point correlation function of the protostars (Fig. 7). For the 37 protostars in the ALMAcovered area, the function is in agreement with a random distribution. However, there are very few short ( $\$ 10000 \mathrm{AU})$ separations, hampering the accuracy of the function; the function is also in agreement with a significant excess of short separations. To better quantify the distribution of protostars in the ISF area, we calculated the two-point correlation function for all 46 protostars in the northern ISF area (see Fig. 1). For this higher number of objects, the uncertainties are smaller and the function indicates a significant excess of separations shorter than $\sim 17000 \mathrm{AU}$ and a marginal excess at separations shorter than roughly $\sim 40000 \mathrm{AU}$. This is somewhat in contradiction with the earlier result that the protostellar cores are in agreement with random distribution. This may result from the small number of protostellar cores in the ALMA-covered region that prohibits detecting a marginal excess of separations. In summary, the data suggest that the dense cores and protostars in the northern ISF show a similar excess of separations between $~ 6000-17000$ AU.

The two-point correlation function of the 51 stars with disks within the ALMA-covered region is consistent with zero at almost all separations (there is a marginally significant positive peak at $\sim 5000 \mathrm{AU}$, see Fig. 7). This indicates that the separation distribution of disks in the region is in agreement with a random distribution, and therefore, it is significantly different from the distributions of the ALMA dense cores and protostars in the northern ISF.

\section{Discussion}

Here, we first discuss the fragmentation picture implied by the observed separation statistics of the different object classes (Sect. 4.1). Then, we discuss the picture in the context of theoretical gravitational fragmentation models (Sect. 4.2).

\subsection{The fragmentation picture implied by the spatial distribution of the cores, protostars, and disks}

Most crucially, we detect increasing grouping of ALMA dense cores at separations below $\sim 17000 \mathrm{AU}$, and especially below $\sim 6000$ AU. We also show that the frequency of short nearest neighbour separations of the cores increases down to our resolution limit (1200 AU); in other words, we do not detect any peaks in the nearest neighbour separation indicating well-defined preferential separations. These observations directly point towards hierarchical, scale-dependent fragmentation of the ISF. The continuous increase of short separations is especially interesting for the models of multiplicity during star formation; numerical 

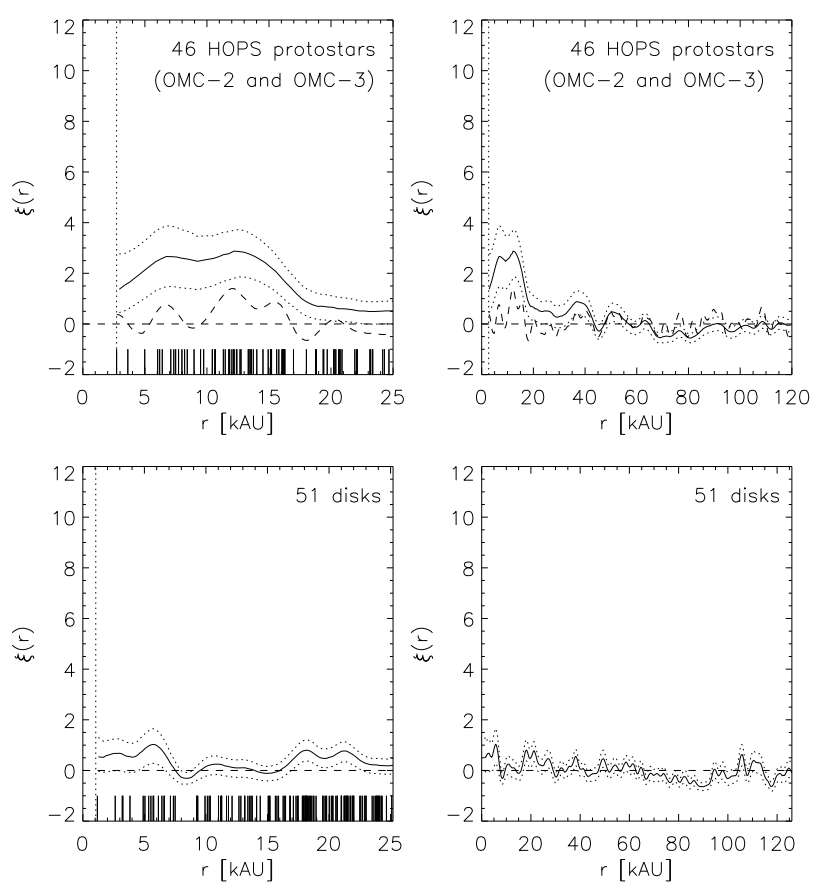

Fig. 7. Two-point correlation function of the separations of the protostars and stars with disks. The frames on the left show the separations until $25 \mathrm{kAU}$ and the frames on the right until $125 \mathrm{kAU}$. The dotted curves show the confidence intervals given by three times the $25 \%$ and $75 \%$ quartiles. The vertical dotted line shows the minimum separation. The dashed horizontal line, drawn at zero, indicates a random distribution. The short vertical lines at the bottom of the panels show the observed separations (only in the panels on the left). The frames for the protostars show the function combining the protostars from the OMC2 and 3 regions with a solid line. The function obtained for the region mapped by ALMA is shown with a dashed line (for visibility, the confidence intervals are not shown).

simulations predict that one efficient formation mechanism of bound multiple systems is core fragmentation at scales between 500-5000 AU (e.g., Offner et al. 2010). Especially, pairs formed at separations below roughly 3000-4000 AU are likely to result in a gravitationally bound multiple system. The strong increase of short core separations we detect below $~ 6000$ AU may mark the regime of these systems (see, e.g., Pineda et al. 2015, for a discovery of a bound core system at these scales).

Importantly, the strong grouping of cores below $6000 \mathrm{AU}$ is driven by the starless cores, while the protostellar cores do not show such a grouping signature (cf., Fig. 6). One should keep in mind the caveats regarding the core identification that may be significant especially for the starless cores (cf., Sect. 2.2). The majority of the starless cores are located close to ( $\$ 5000 \mathrm{AU})$ protostellar cores, indicating a possibility that some may be column density enhancements caused by, e.g., outflow cavities. However, the effect of possible misclassifications to the twopoint correlation analysis is likely not strong (see Appendix C). With these caveats in mind, we speculate about the possible origin of the grouping difference between the protostellar and starless cores. One possibility is an evolutionary sequence in which the protostellar cores have lost some of their grouping inherited from the fragmentation process, but this signature is still imprinted in the grouping of starless cores. Other hypotheses could be that not all cores end up forming stars or that the cores merge during their evolution. Further studies on the kinematics of the
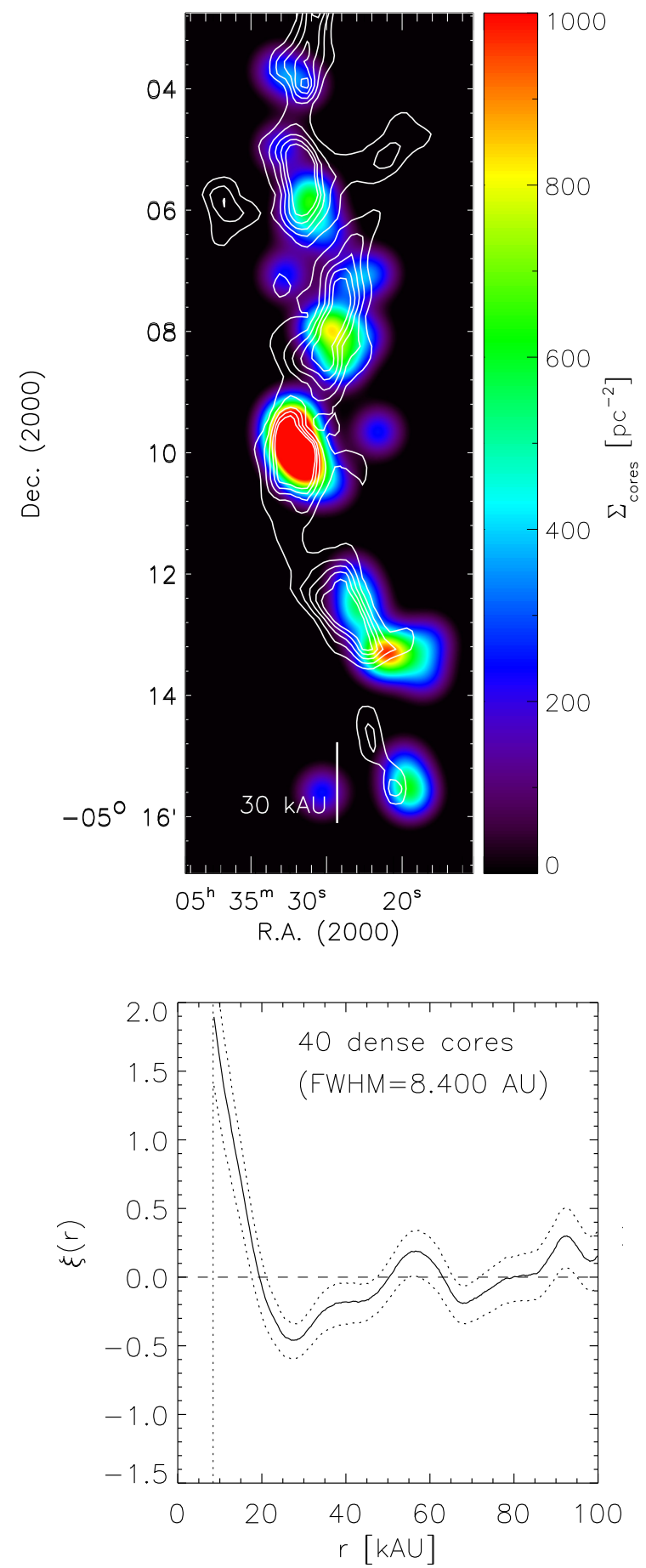

Fig. 8. Top: surface number density of the dense cores identified from the $3 \mathrm{~mm}$ ALMA continuum data. The white contours show the Herschel-derived column densities at $N(\mathrm{H})=\{60,80,100,120,140\} \times$ $10^{21} \mathrm{~cm}^{-2}$ (Stutz \& Kainulainen 2015). Bottom: two-point correlation function of the 43 dense cores, derived with a smoothing function with $F W H M=20^{\prime \prime}=8400 \mathrm{AU}$.

ALMA-detected cores could address the strength of the increase in core separations below $\sim 6000 \mathrm{AU}$ and explore the origin of it.

Previously, Teixeira et al. (2016) have studied a section of the ISF in OMC-1n and claimed evidence of two preferred nearest-neighbour core separation scales, at $5500 \mathrm{AU}$ and $13000 \mathrm{AU}$. Our data does not indicate peaks in the preferred core separations, but rather a continuous increase of short separations. This difference may be due to various reasons. For example, 
Teixeira et al. (2016) only had a sample of 24 cores, hampering the operation of statistical estimators; they do not present an analysis of how significant their detections of the preferred scales are. Also, our core identification method is different from that of Teixeira et al. (2016), making a direct comparison difficult. Studies targeting other massive filaments have commonly quantified mean separations between structures detected at different spatial scales (e.g., Jackson et al. 2010; Miettinen 2012; Kainulainen et al. 2013; Wang et al. 2014; Beuther et al. 2015a; Contreras et al. 2016). However, none of these works to our knowledge has analysed the higher-order statistics of the fragment separations and linked them to the young stellar distribution in the clouds.

Our analysis of the core distribution has so far concentrated on the small scales. We now consider the distribution at the scales larger than the grouping identified by the two-point correlation analysis ( $>17000 \mathrm{AU})$. Figure 8 shows the number of detected ALMA cores per unit area, derived using a Gaussian smoothing kernel that has the FWHM of 40" (17000 AU). The distribution is clearly not random, but concentrated on groups along the filament. The groups are roughly $30000 \mathrm{AU}$ in size and they are separated quasi-periodically by roughly $50000 \mathrm{AU}$ $(62000 \pm 19000 \mathrm{AU}$ when considering the six largest groups and $42000 \pm 26000 \mathrm{AU}$ if considering the feature at around Dec $\approx-5.13$ m.00 as two groups). The core groups coincide with the local maxima of dust column density as probed by Herschel that have the mean separation of $56000 \pm 14000$ AU (see Fig. 8). Thus, the ALMA and Herschel data provide independent measurements of the quasi-periodic fragmentation mode and they indicate that the neighbouring cores have common, tenth-of-aparsec-scale envelopes.

The quasi-periodic fragmentation mode can also be inferred from the two-point correlation function of the cores ALMA. We looked for signals in the function at large scales by heavily smoothing it to gain a better signal-to-noise. The function derived with a smoothing function that has $F W H M=20^{\prime \prime}=$ $8400 \mathrm{AU}$ is shown in Fig. 8. The function shows a significant negative feature between $~ 20000-40000$ AU, indicating a deficit of cores at these separations with respect to a random distribution (note that by definition, the two-point correlation function cannot have values lower than -1 , see Eq. (4)). The negative feature is followed by a marginally significant peak at $\sim 55000$ AU. We show in Appendix B that marginally significant negative and positive signatures are expected for periodically grouped cores at the characteristic size of the groups and at the wavelength of the grouping period, respectively.

In summary, the spatial distribution of the ALMA-detected cores indicates periodic grouping into core groups roughly $30000 \mathrm{AU}$ in size, separated by roughly $55000 \mathrm{AU}$. At scales smaller than $30000 \mathrm{AU}$, i.e., inside the individual groups, the cores group below about $17000 \mathrm{AU}$ and especially strongly below 6000 AU (cf., Fig. 6).

The above picture is in agreement with previous studies that have found signatures interpreted as co-existing spherical and cylindrical gravitational fragmentation modes in highly super-critical filaments, with the transition between the two occurring at a few tenths of a parsec $(0.3 \mathrm{pc}=60000 \mathrm{AU}$; Kainulainen et al. 2013; Takahashi et al. 2013). These works have suggested that the transition could result, for example, from the finite-size effects in filaments: below a certain sizescale local collapse may proceed faster than the global collapse of the filament (e.g., Pon et al. 2011; Kainulainen et al. 2013). Regardless of its driver, our data suggests that a similar signature is detectable in the separation distribution of the cores: the $55000 \mathrm{AU}$ separations between the groups correspond to the scale of the filamentary fragmentation mode, and the increasing separations below $\sim 17000$ AU correspond to the scale of the spherical fragmentation mode. We further address the feasibility of this interpretation in Sect. 4.2.

The relationship between the separation distributions of the ALMA dense cores and HOPS protostars and disks can indicate temporal evolution. The dense core and protostar separation distributions show similarity between 6000-17000 AU. This suggests that the initial grouping of the ALMA cores is not totally erased over the core life-time, nor the protostar lifetime ( 0.5 Myr; e.g., Dunham et al. 2014). However, the different grouping of stars with disks shows that it is erased over the life-time of stars with disks ( 2 Myr; e.g., Evans et al. 2009; Dunham et al. 2014). This provides support for the dynamical slingshot model of Stutz \& Gould (2016), in which the gravitational potential well of the surrounding gas reservoir in the ISF holds the protostars close to their maternal filament section. However, the (proto-)stars decouple from the gas reservoir due to the slingshot, at which point they can loose their initial grouping properties. We note a general caveat that our data probe a narrow field centred on the densest part of the filament. The disk population extends to a significantly larger volume than this field, occupying also a larger volume than dense cores and protostars do. We are insensitive to the grouping signatures the disk population may show outside this field.

\subsection{Applicability of gravitational fragmentation models}

We now discuss the observed fragmentation of the ISF in the context of analytic, idealised gravitational fragmentation models. First, shortly recall the physical properties of the ISF. The line-mass of the ISF is $385 \times(D / \mathrm{pc})^{3 / 8} M_{\odot} \mathrm{pc}^{-1}$, where $D$ is the width of the region that is included in the mass calculation. The radial density structure of the ISF is proportional to $r^{-13 / 8}$ (Stutz \& Gould 2016). The CO line emission from the ISF shows linewidths around $3-4 \mathrm{~km} \mathrm{~s}^{-1}$ and the $\mathrm{C}^{18} \mathrm{O}$ emission around $1 \mathrm{~km} \mathrm{~s}^{-1}$ when averaged over a threearcminute beam (Nishimura et al. 2015); this indicates strong non-thermal motions given the typical temperatures of $\sim 20 \mathrm{~K}$ (e.g., Stutz \& Kainulainen 2015). The Zeeman-splitting measurements suggest that the filament is wrapped in a helical magnetic field (Heiles 1997, see also discussion in Stutz \& Gould 2016). In this paper, we have shown that the filament is fragmented hierarchically into groups of dense cores and that the fragmentation within these groups increases down to $1000 \mathrm{AU}$ scales.

Consider now the gravitational fragmentation models for self-gravitating, non-magnetized, infinitely long, equilibrium cylinders in the regime of small perturbations (e.g., Ostriker 1964; Nagasawa 1987; Inutsuka \& Miyama 1992; Fiege \& Pudritz 2000b; Fischera \& Martin 2012). These models have established that there is a range of unstable wavelengths that lead to growing perturbations. Within this range the perturbation growth rate has a unique maximum that defines the fastest-growing wavelength. Similarly in the spherical case, gravitational fragmentation is expected at the Jeans' length scale (Jeans 1929), provided that sufficiently strong initial density fluctuations are present (e.g., Larson 1985). The fundamental prediction of both cylindrical and spherical models is a well-defined fragmentation scale: the fastest growing perturbation wavelength for cylinders and the Jeans' length for spheres. In both cases, this scale is coupled to the gas density. 
We immediately notice problems in the applicability of the idealised cylindrical fragmentation models. Most pressingly, the ISF is gravitationally super-critical by a factor of roughly 20 , and thus, not near-critical. The applicability of the nearequilibrium cylindrical models to highly super-critical filaments (such as the ISF) has not been established. It has been argued on qualitative grounds, and commonly assumed in literature, that non-thermal motions can provide microturbulent pressure support to super-critical filaments, effectively increasing their critical line-mass (e.g., Fiege \& Pudritz 2000a; Jackson et al. 2010; Fischera \& Martin 2012; Heitsch 2013; Hernandez et al. 2012; Beuther et al. 2015a). However, we need to recognise that the nature of non-thermal motions within highly super-critical filaments (including the ISF) has not been established. Observations of nearby, slightly super-critical filaments whose velocity structure has been scrutinised in high detail do not support the picture of micro-turbulent motions within filaments (Hacar et al. 2013). Recent observations suggest analogous, systematic motions also within highly super-critical filaments (e.g. Henshaw et al. 2014; Beuther et al. 2015a). Furthermore, the radial density distribution of the ISF is $\rho \propto r^{-1.6}$ (Stutz \& Gould 2016), flatter than that of the idealised hydrostatic solution $\left(\rho \propto r^{? 4}\right.$; Ostriker 1964). Theoretical works indicate that near-critical filaments wrapped by helical magnetic fields (Fiege \& Pudritz 2000a), or collapsing filaments with a non-isothermal equation of state (Kawachi \& Hanawa 1998), can have flat radial profiles close to $\rho \propto r^{-2}$. However, the applicability of these models to highly super-critical filaments is not clear. Finally, the fragmentation of the ISF is not in agreement with the fragmentation models of near-critical filaments. The ISF does not show a single fragmentation scale, but a more complicated, multi-scale picture: an increase of short separations below $17000 \mathrm{AU}$, especially below $6000 \mathrm{AU}$, and periodic grouping at $\sim 50000 \mathrm{AU}$.

Given the above, the ability of the currently existing analytic, idealised models to address the fragmentation of highly supercritical filaments such as the ISF is questionable at best. This calls for theoretical works aiming at explaining multi-scale, hierarchical fragmentation. In the following, we compare the predictions of the gravitational fragmentation models with the observed fragmentation pattern of the ISF with this short-coming in mind.

\subsection{Comparison against gravitational fragmentation models}

In the absence of a theory well-suited for highly super-critical filaments we calculate the predictions of the spherical and cylindrical gravitational fragmentation models for the fragmentation scale of the ISF. Spherical gravitational fragmentation predicts preferential fragmentation at the Jeans' length-scale, $l_{\mathrm{J}}=$ $c_{\mathrm{s}}(G / \bar{\rho})^{1 / 2}$, where $c_{\mathrm{s}}$ is the isothermal sound speed. To evaluate this, we adopt the temperature of $20 \mathrm{~K}$ as before. Stutz \& Gould (2016) have evaluated the (cylindric) density profile of the ISF to be $17 M_{\odot} \mathrm{pc}^{-3}(r / \mathrm{pc})^{-13 / 8}$ down to their resolution limit of $0.04 \mathrm{pc}$. If we use the density at $0.04 \mathrm{pc}$ as the proxy of the density relevant for Jeans' fragmentation, the density of $\sim 10^{5} \mathrm{~cm}^{-3}$ and the fragmentation scale of $\sim 10000 \mathrm{AU}$ follows. We also make another estimate of the relevant density by estimating the mean densities of the six main structures in the Herschel-derived column density map of the area (see Fig. 8). We do this by summing up all column densities in the area of the structures and assuming the structures are spherical. This resulted in the mean density of $2 \times 10^{5} \mathrm{~cm}^{-3}$, and from therein, in the fragmentation scale of $\sim 7000 \mathrm{AU}$. These fragmentation scales are similar to the size scales over which the cores show an excess of separations, i.e., $<17000$ AU. The scales are smaller by factors of six and eight compared to the 55000 AU separation between the core groups identified from the ALMA data (or the separation between the dust structures identified from the Herschel data, cf., Fig. 8). Thus, from this point-of-view, the fragmentation at $\sim 10000 \mathrm{AU}$ scales is in a rough agreement with the prediction of Jeans' fragmentation, while the fragmentation at larger scales seems less likely to be.

What is the prediction of the cylindrical fragmentation models? In the gravitational fragmentation of a near-equilibrium filament (i.e., cylinder), the wavelength of the fastest growing unstable mode depends on the FWHM of the filament, or equivalently, on the central density, scale-radius, or linemass (these are all inter-connected in the equilibrium solution). The fragmentation scale is then $\sim 3$ times the FWHM of the filament (Fischera \& Martin 2012, see also Nagasawa 1987; Inutsuka \& Miyama 1992; Fiege \& Pudritz 2000b). We do not know well the FWHM of the ISF, but we can use the dependency of the FWHM on central density to obtain an estimate. For a near-equilibrium filament, $F W H M \approx 4 \times c_{\mathrm{s}}\left(4 \pi G \rho_{\mathrm{c}}\right)^{-0.5}$ (Fischera \& Martin 2012). If we again use the density at $r=$ $0.04 \mathrm{pc}$ as the relevant density, the $F W H M \approx 0.06 \mathrm{pc}$ follows, yielding the fragmentation scale of $\sim 40000$ AU. If we use the density derived from Herschel data (as above), the scale is $\sim 30000$ AU. These are within a factor of 2 of the periodic separation between the ALMA-detected core groups, or between the Herschel-detected dust structures ( 55000 AU). Thus, one can speculate that filamentary gravitational fragmentation may be one process affecting the fragmentation at these scales, even if the models do not capture all relevant physical processes affecting fragmentation. Several other works have also found fragmentation in highly-supercritical filaments at these scales (e.g., Busquet et al. 2013; Kainulainen et al. 2013; Takahashi et al. 2013; Wang et al. 2014; Beuther et al. 2015b; Teixeira et al. 2016; Henshaw et al. 2016). One should note that, e.g., the presence of magnetic fields can significantly alter the prediction for the fragmentation scale (e.g., Fiege \& Pudritz $2000 \mathrm{~b}$ ). We also have assumed an inclination angle of zero throughout this paper.

In summary, the observational picture of fragmentation within ISF is not predicted by any single existing fragmentation model alone, even if the models capture some characteristics of the observed fragmentation. To make progress, a multiscale model specific for highly super-critical filaments is needed. Since the models for near-critical filaments are partially in agreement with observations, they may provide a reasonable basis for such models. We can speculate about such framework in the light of the fragmentation time-scales predicted by the existing models. A high-aspect ratio filament first fragments following the prediction of the cylindrical gravitational fragmentation model (in the ISF, this would correspond to the fragmentation seen at 50000 AU scale). The characteristic time-scale for this is $\tau_{\mathrm{m}} \approx$ $3 \times \sqrt{1 /\left(4 \pi \rho_{\mathrm{c}} G\right)} \approx 0.2 \mathrm{Myr}$. By definition, the fragments have a higher mean density than the initial filament (assuming no mass is lost to stars at this point). This leads to a shorter fragmentation time-scale within the fragment compared to the initial fragmentation time, by a factor of $\rho_{\mathrm{c}}^{-1 / 2}$ (e.g., Fiege \& Pudritz 2000b; Fischera \& Martin 2012). If the global collapse time-scale of the fragments is their free-fall time (about $0.1 \mathrm{Myr}$ at the density of $10^{5} \mathrm{~cm}^{-3}$ ), this leads to a competition between the global collapse of the fragment and further fragmentation inside it (e.g., Pon et al. 2011, 2012; Clarke \& Whitworth 2015). Clearly, the latter must ensue faster, because we observe fragmentation down 
to $\sim 1000$ AU scales. However, it is not obvious how to arrive to this conclusion, because both the free-fall time and the fragmentation time depend on density with the same power $\left(\propto \rho^{-0.5}\right)$. One possible route is provided by magnetic fields that may provide support against a global collapse while not strongly affecting the fragmentation time-scale (e.g., Fiege \& Pudritz 2000a,b, see also Seifried \& Walch 2015, for a numerical study showing an analogous effect in filaments that are thermally near-critical). Another possibility could be large-enough, pre-existing density fluctuations that can grow and collapse faster than the global, longitudinal collapse ensues (Larson 1985; Pon et al. 2011). Supporting this possibility, filaments with low star formation activity have been observed to contain significant density fluctuations (e.g., Roy et al. 2015; Kainulainen et al. 2016). Developing this cartoon framework into a coherent theory is beyond this paper, but it would be a crucial topic for future works that aim at understand the fragmentation of highly super-critical filaments.

\section{Conclusions}

We present a fragmentation study of a $1.6 \mathrm{pc}$ long section of the ISF in the Orion A molecular cloud, specifically covering the OMC-2 and a part of the OMC-3 region. We employ ALMA $3 \mathrm{~mm}$ continuum emission data that reach the spatial resolution of $\sim 3^{\prime \prime}$, or $1200 \mathrm{AU}$. We also study the relationship between the distributions of the ALMA-detected dense cores, protostars, and stars with disks using the census of the young stellar population built by the Spitzer Orion Survey and HOPS. Our conclusions are as follows.

1. The ALMA data reveal numerous substructures from the ISF, including compact and extended structures and filaments. Most of the single-peaked structures detected previously in coarser resolution resolve into substructures. Especially, we detect a prominent filament in the FIR 3-5 region connecting a series of dense cores and protostars.

2. We identify 43 dense cores from the ALMA data, $26(60 \%)$ of which coincide with protostars and $17(40 \%)$ of which are starless. The nearest neighbour separation distribution of the ALMA cores increases down to our resolution limit, indicating that there is no preferential nearest-neighbour separation. The nearest-neighbour separation distributions of the starless cores and protostellar cores are different; the starless cores show a higher fraction of short nearest-neighbour separations than the protostellar cores.

3. The two-point correlation analysis of the dense core separations shows that the ALMA cores are significantly grouped at separations smaller than $\sim 17000$ AU and strongly grouped at separations smaller than $\sim 6000 \mathrm{AU}$. This is different for starless and protostellar cores: the starless cores show strong grouping, while the protostellar cores show only marginally significant grouping, however, the grouping strength of the cores (especially starless cores) may be affected by caveats in the core identification. The increase of grouping below $6000 \mathrm{AU}$ may be related to the regime where interactions between the cores become abundant. The spatial distribution of the cores also indicates a quasi-periodic grouping of the cores into groups $\sim 30000 \mathrm{AU}$ in size, separated by $\sim 50000$ AU. These groups corresponds to the gas morphology traced by the Herschel column density map of the region (Stutz \& Kainulainen 2015).

4. The two-point correlation analysis shows that the HOPS protostars in the entire northern ISF are significantly grouped at separations shorter than $\sim 17000$ AU. Thus, they show partially similar grouping as the dense cores; however, the protostars do not show strong grouping below $~ 6000$ AU like the dense cores do. The distribution of stars with disks is in agreement with random distribution within the ALMAcovered region, and it is thus significantly different from that of the ALMA cores and protostars. These results suggest that the grouping of dense cores, resulting from the fragmentation process, is not totally erased during the protostar life-time, but it is erased during the longer life-times of stars with disks. This is in agreement with the picture of (Stutz \& Gould 2016) in which the protostars are ejected from the filament by the slingshot mechanism.

5. The hierarchical, scale-dependent fragmentation we observe in the ISF is not self-consistently predicted by any existing gravitational fragmentation models. We use the density profile along the filament derived by Stutz \& Gould (2016) and show that the predictions for the Jeans' fragmentation scale is $\sim 10000 \mathrm{AU}$ and for the filamentary gravitational fragmentation scale is $\sim 40000$ AU. These scales are similar to the scales at which the cores show grouping, suggesting that gravitational fragmentation is an important process, even though our understanding of how exactly it proceeds is incomplete.

Our results provide the most sensitive view of the $1000 \mathrm{AU}$ scale fragmentation within a massive filament to date. Especially, our detection of increasingly abundant separations below $6000 \mathrm{AU}$ opens the question of the abundance of bound groups at these separations. Further observations targeting the dynamics of the cores and scales between 500-1000 AU will be able to quantify this and address the question of stellar multiplicity resulting from core fragmentation. Similarly, our work highlights the need for theoretical work addressing the structure of highly super-critical filaments and the multi-scale nature of their fragmentation; our observations provide concrete constraints for testing such models.

Acknowledgements. The authors are grateful to Ralph Pudritz and Jaime Piñeda for fruitful discussions. The work of J.K. and A.S. was partially supported by the Deutsche Forschungsgemeinschaft priority program 1573 ("Physics of the Interstellar Medium"). This project has received funding from the European Union's Horizon 2020 research and innovation programme under grant agreement No 639459 (PROMISE). This paper makes use of the following ALMA data: ADS/JAO.ALMA\#2013.1.01114.S. ALMA is a partnership of ESO (representing its member states), NSF (USA) and NINS (Japan), together with NRC (Canada), NSC and ASIAA (Taiwan), and KASI (Republic of Korea), in cooperation with the Republic of Chile. The Joint ALMA Observatory is operated by ESO, AUI/NRAO and NAOJ.

\section{References}

Adams, J. D., Herter, T. L., Osorio, M., et al. 2012, ApJ, 749, L24 Abreu-Vicente, J., Ragan, S., Kainulainen, J., et al. 2016, A\&A, 590, A131 Alves, J. F., Lada, C. J., \& Lada, E. A. 2001, Nature, 409, 159

Alves de Oliveira, C., Schneider, N., Merín, B., et al. 2014, A\&A, 568, A98 André, P., Di Francesco, J., Ward-Thompson, D., et al. 2014, in Protostars and Planets VI (Tucson: University of Arizona Press), 27

Bally, J., Langer, W. D., Stark, A. A., \& Wilson, R. W. 1987, ApJ, 312, L45 Beaumont, C. N., Offner, S. S. R., Shetty, R., et al. 2013, ApJ, 777, 173 Bergin, E. A., \& Tafalla, M. 2007, ARA\&A, 45, 339

Berry, D. S., Reinhold, K., Jenness, T., \& Economou, F. 2007, Astronomical Data Analysis Software and Systems XVI, 376, 425

Beuther, H., Henning, T., Linz, H., et al. 2010, A\&A, 518, L78

Beuther, H., Ragan, S. E., Johnston, K., et al. 2015a, A\&A, 584, A67

Beuther, H., Henning, T., Linz, H., et al. 2015b, A\&A, 581, A119

Busquet, G., Zhang, Q., Palau, A., et al. 2013, ApJ, 764, L26

Chini, R., Reipurth, B., Ward-Thompson, D., et al. 1997, ApJ, 474, L135

Clarke, S. D., \& Whitworth, A. P. 2015, MNRAS, 449, 1819 
Contreras, Y., Garay, G., Rathborne, J. M., \& Sanhueza, P. 2016, MNRAS, 456, 2041

di Francesco, J., Evans, N. J., II, Caselli, P., et al. 2007, in Protostars and Planets V (Tucson: University of Arizona Press), 17

Dunham, M. M., Stutz, A. M., Allen, L. E., et al. 2014, in Protostars and Planets VI (Tucson: University of Arizona Press), 195

Evans, N. J., II, Dunham, M. M., Jørgensen, J. K., et al. 2009, ApJS, 181, 321

Fiege, J. D., \& Pudritz, R. E. 2000a, MNRAS, 311, 85

Fiege, J. D., \& Pudritz, R. E. 2000b, MNRAS, 311, 105

Fischera, J., \& Martin, P. G. 2012, A\&A, 542, A77

Furlan, E., Megeath, S. T., Osorio, M., et al. 2014, ApJ, 786, 26

Furlan, E., Fischer, W. J., Ali, B., et al. 2016, ApJS, 224, 5

Gutermuth, R. A., Pipher, J. L., Megeath, S. T., et al. 2011, ApJ, 739, 84

Hacar, A., Tafalla, M., Kauffmann, J., \& Kovács, A. 2013, A\&A, 554, A55

Heiles, C. 1997, ApJS, 111, 245

Heitsch, F. 2013, ApJ, 769, 115

Henning, T., Linz, H., Krause, O., et al. 2010, A\&A, 518, L95

Henshaw, J. D., Caselli, P., Fontani, F., Jiménez-Serra, I., \& Tan, J. C. 2014, MNRAS, 440, 2860

Henshaw, J. D., Caselli, P., Fontani, F., et al. 2016, MNRAS, 463, 146

Hernandez, A. K., Tan, J. C., Kainulainen, J., et al. 2012, ApJ, 756, L13

Hill, T., Motte, F., Didelon, P., et al. 2011, A\&A, 533, A94

Inutsuka, S.-I., \& Miyama, S. M. 1992, ApJ, 388, 392

Inutsuka, S.-I., \& Miyama, S. M. 1997, ApJ, 480, 681

Jackson, J. M., Finn, S. C., Chambers, E. T., et al. 2010, ApJ, 719, L185

Jeans, J. H. 1929, Astronomy and Cosmogony (Cambridge University Press, reprinted by Dover, New York, 1961)

Johnstone, D., \& Bally, J. 1999, ApJ, 510, L49

Kainulainen, J., Lehtinen, K., Väisänen, P., Bronfman, L., \& Knude, J. 2007, A\&A, 463, 1029

Kainulainen, J., Lada, C. J., Rathborne, J. M., \& Alves, J. F. 2009, A\&A, 497, 399

Kainulainen, J., Ragan, S. E., Henning, T., \& Stutz, A. 2013, A\&A, 557, A120

Kainulainen, J., Hacar, A., Alves, J., et al. 2016, A\&A, 586, A27

Kandori, R., Nakajima, Y., Tamura, M., et al. 2005, AJ, 130, 2166

Kawachi, T., \& Hanawa, T. 1998, PASJ, 50, 577

Landy, S. D., \& Szalay, A. S. 1993, ApJ, 412, 64

Lane, J., Kirk, H., Johnstone, D., et al. 2016, ApJ, 833, 44
Larson, R. B. 1985, MNRAS, 214, 379

Li, D., Kauffmann, J., Zhang, Q., \& Chen, W. 2013, ApJ, 768, L5

Megeath, S. T., Gutermuth, R., Muzerolle, J., et al. 2012, AJ, 144, 192 Megeath, S. T., Gutermuth, R., Muzerolle, J., et al. 2016, AJ, 151, 5

Meingast, S., Alves, J., Mardones, D., et al. 2016, A\&A, 587, A153 Mezger, P. G., Zylka, R., \& Wink, J. E. 1990, A\&A, 228, 95

Miettinen, O. 2012, A\&A, 540, A104

Nagasawa, M. 1987, Prog. Theor. Phys., 77, 635

Nishimura, A., Tokuda, K., Kimura, K., et al. 2015, ApJS, 216, 18

Offner, S. S. R., Kratter, K. M., Matzner, C. D., et al. 2010, ApJ, 725, 1485

Ostriker, J. 1964, ApJ, 140, 1056

Peterson, D. E., \& Megeath, S. T. 2008, in Handbook of Star Forming Regions, Vol. I, ed. B. Reipurth, 590

Pillai, T., Wyrowski, F., Menten, K. M., \& Krügel, E. 2006, A\&A, 447, 929

Pineda, J. E., Offner, S. S. R., Parker, R. J., et al. 2015, Nature, 518, 213

Pon, A., Johnstone, D., \& Heitsch, F. 2011, ApJ, 740, 88

Pon, A., Toalá, J. A., Johnstone, D., et al. 2012, ApJ, 756, 145

Ragan, S. E., Henning, T., Tackenberg, J., et al. 2014, A\&A, 568, A73

Roy, A., André, P., Arzoumanian, D., et al. 2015, A\&A, 584, A111

Sadavoy, S. I., Stutz, A. M., Schnee, S., et al. 2016, A\&A, 588, A30

Schlafly, E. F., Green, G., Finkbeiner, D. P., et al. 2014, ApJ, 786, 29

Schneider, N., Csengeri, T., Hennemann, M., et al. 2012, A\&A, 540, L11

Seifried, D., \& Walch, S. 2015, MNRAS, 452, 2410

Shimajiri, Y., Takahashi, S., Takakuwa, S., et al. 2008, ApJ, 683, 255

Shimajiri, Y., Takahashi, S., Takakuwa, S., Saito, M., \& Kawabe, R. 2009, PASJ, 61,1055

Shimajiri, Y., Sakai, T., Kitamura, Y., et al. 2015, ApJS, 221, 31

Smith, R. J., Clark, P. C., \& Bonnell, I. A. 2008, MNRAS, 391, 1091

Stutzki, J., \& Guesten, R. 1990, ApJ, 356, 513

Stutz, A. M., \& Gould, A. 2016, A\&A, 590, A2

Stutz, A. M., \& Kainulainen, J. 2015, A\&A, 577, L6

Stutz, A., Launhardt, R., Linz, H., et al. 2010, A\&A, 518, L87

Stutz, A. M., Tobin, J. J., Stanke, T., et al. 2013, ApJ, 767, 36

Takahashi, S., Ho, P. T. P., Teixeira, P. S., Zapata, L. A., \& Su, Y.-N. 2013, ApJ, 763, 57

Teixeira, P. S., Takahashi, S., Zapata, L. A., \& Ho, P. T. P. 2016, A\&A, 587, A47

Tobin, J. J., Stutz, A. M., Megeath, S. T., et al. 2015, ApJ, 798, 128

Wang, K., Zhang, Q., Testi, L., et al. 2014, MNRAS, 439, 3275 

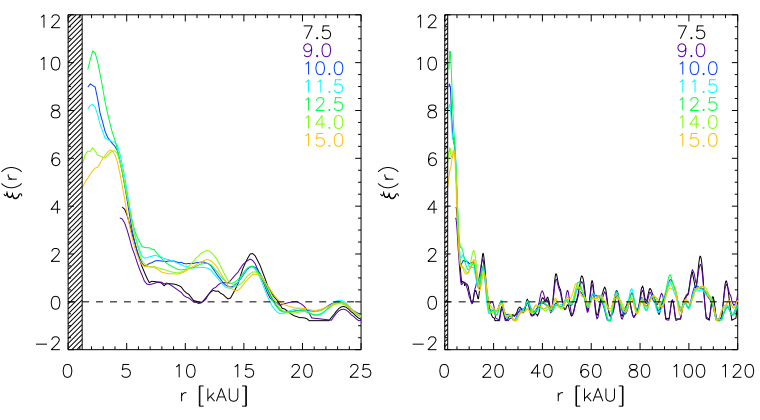

Fig. A.1. Two-point correlation functions of the dense cores populations identified with different background filter widths of the gaussclumps algorithm. The left panel shows the function between 0-25 kAU and the right panel between 0-120 kAU. The coloured numbers show the background filter width, in arcseconds, used in the core identification.

\section{Appendix A: Effect of the background filter width on the core identification}

We tested the effect of the background filter width used in the dense core identification on the resulting two-point correlation functions. We repeated the core identification with the filter widths between $7.5^{\prime \prime}-15^{\prime \prime}$. The resulting two-point correlation functions are shown in Fig. A.1. The functions are drawn only above the minimum separation of the dense cores. The test shows that the choice of the background filter does not affect the main conclusions derived from the two-point correlation functions. This comes from the fact that the majority of the cores are identified similarly regardless of the choice of the background filter width. When a small filter is chosen, only the strongest (most significant) cores are detected. When the filter width approaches $15^{\prime \prime}$, structures identified as separate dense cores are occasionally identified as one, larger core. The test shows that optimum filter width for detecting cores in $3^{\prime \prime}$ resolution is between $\sim 7.5^{\prime \prime}-12.5^{\prime \prime}$. Our final choice, $10^{\prime \prime}$, is within this range.

\section{Appendix B: Two-point correlation analysis at large scales}

We estimated the significance of the quasi-periodic core grouping at 50000 scales with a simple simulation. The simulation setup was guided by the surface number density of the ALMAdetected cores (Fig. 8). We construct a simplistic model in which groups of cores are located along a line with a constant separation. The simulation area matches the dimensions of the ALMA field, and we place six groups in the field. The groups have the size of $42000 \mathrm{AU}$ and their separation is $56000 \mathrm{AU}$. The placement of cores within the groups is random. The bottom right panel of Fig. 8 shows a typical two-point correlation function resulting for 43 simulated cores. The simulation illustrates that the basic signatures of this configuration, i.e., the deficit of cores at $D(42000 \mathrm{AU})$ and the excess at $\lambda(56000 \mathrm{AU})$, are generally expected to be detectable with a sample of 43 cores, albeit with a low significance.

\section{Appendix C: On the robustness of the two-point correlation analysis}

The continuum data we exploit in this data does not alone allow disentangling cores and core-like objects (e.g., walls of outflow cavities, structures overlapping along the line-of-sight) from each others. Consequently, especially the weakest structures classified as starless cores may be misclassifications. To

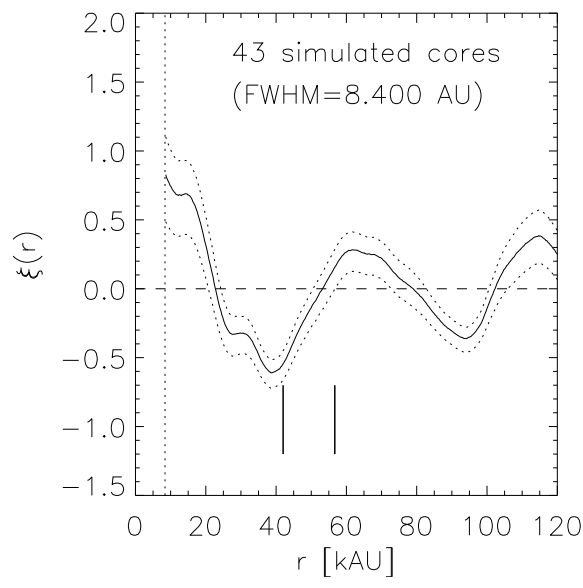

Fig. B.1. Two-point correlation function of a simulation in which 43 cores are randomly placed in six groups along a line. The diameter of the groups is $42000 \mathrm{AU}$ and their separation $56000 \mathrm{AU}$. These distances are indicated with vertical lines. The function was derived with a smoothing function that has $F W H M=20^{\prime \prime}=8400 \mathrm{AU}$.
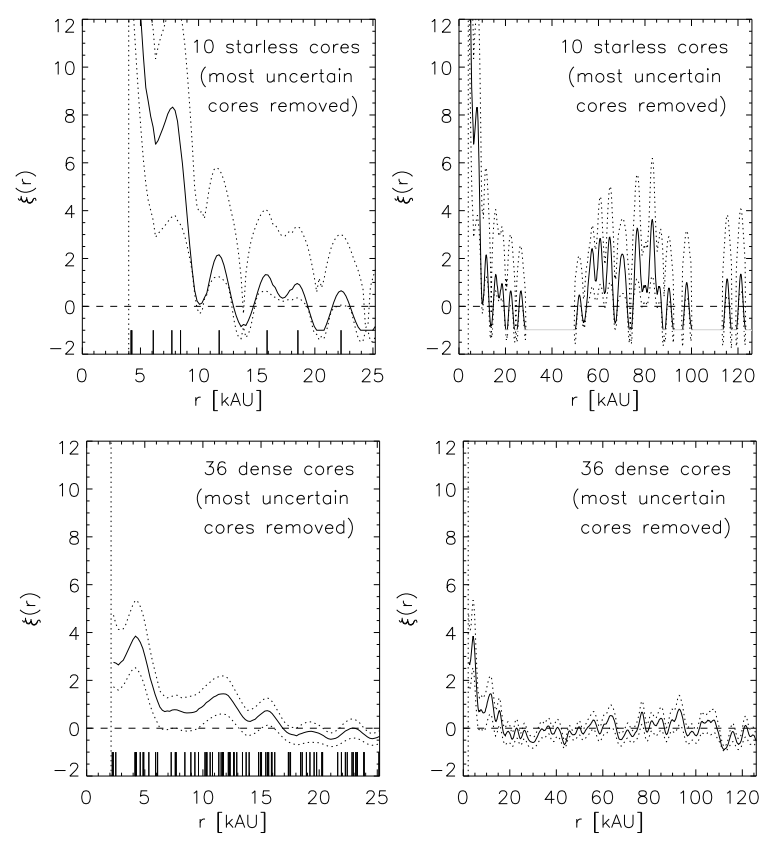

Fig. C.1. Two-point correlation function of the separations of all ALMA dense cores and starless cores, excluding the seven most uncertain starless cores. In the top right panel, there are not enough separations to compute the two-point correlation function at all scales.

address the effect of this possibility on the two-point correlation analysis, we derived the two-point correlation functions of starless cores and all dense cores excluding seven weakest starless cores $(\# 5,15,18,19,23,26,35)$. The resulting two-point correlation functions are shown in Fig. C.1. The two-point correlation function for starless cores only includes ten cores and is very uncertain. However, it do shows a significant excess of short separations $(\$ 10000 \mathrm{AU})$. The two-point correlation function for all dense cores shows significant excess of separations below $\sim 15000 \mathrm{AU}$. The function increases down to our resolution limit, albeit less strongly as when all identified dense cores are included (Fig. 6). We conclude that the results based on twopoint correlation analysis are not strongly affected by possible misclassifications of low-intensity starless cores. 